ALEA, Lat. Am. J. Probab. Math. Stat. 17, 825-855 (2020)

DOI: $10.30757 /$ ALEA.v17-32

\title{
Two limit theorems for the high-dimensional two-stage contact process
}

\section{Xiaofeng Xue}

School of Science, Beijing Jiaotong University

No.3 Shangyuancun, Haidian District,

Beijing, China.

E-mail address: xfxue@bjtu.edu.cn

URL: http://faculty.bjtu.edu.cn/8975/

Abstract. In this paper we are concerned with the two-stage contact process introduced by Krone (1999) on a high-dimensional lattice. By comparing this process with an auxiliary model which is a linear system, we obtain two limit theorems for this process as the dimension of the lattice grows to infinity. The first theorem is about the upper invariant law of the process. The second theorem is about asymptotic behavior of the critical value of the process. These two theorems can be considered as extensions of their counterparts for the basic contact processes proved by Griffeath (1983) and Schonmann and Vares (1986).

\section{Introduction}

In this paper we are concerned with the two-stage contact process on $\mathbb{Z}^{d}$ introduced in Krone (1999) authored by Krone. First we introduce some notation and definitions for later use. For each

$$
x=(x(1), \ldots, x(d)) \in \mathbb{Z}^{d},
$$

we use $\|x\|$ to denote the $l_{1}$-norm of $x$, i.e., $\|x\|=\sum_{i=1}^{d}|x(i)|$. For any $x, y \in \mathbb{Z}^{d}$, we write $x \sim y$ when and only when $\|x-y\|=1$. In other words, $x \sim y$ means that $x$ and $y$ are neighbors on $\mathbb{Z}^{d}$. For $1 \leq i \leq d$, we use $e_{i}$ to denote the $i$ th elementary unit vector of $\mathbb{Z}^{d}$, i.e.,

$$
e_{i}=(0, \ldots, 0, \underset{i \mathrm{th}}{1}, 0, \ldots, 0) .
$$

We use $O$ to denote the origin of $\mathbb{Z}^{d}$, i.e., $O=(0,0, \ldots, 0)$.

Received by the editors February 2nd, 2019; accepted August 1st, 2020.

2010 Mathematics Subject Classification. 60K35.

Key words and phrases. Two-stage, contact process, critical value, invariant measure.

Research supported by the National Natural Science Foundation of China. 
The two-stage contact process $\left\{\eta_{t}\right\}_{t \geq 0}$ on $\mathbb{Z}^{d}$ is a continuous-time Markov process with state space $X=\{0,1,2\}^{Z^{d}}$ and generator $\Omega$ given by

$$
\Omega f(\eta)=\sum_{x \in \mathbb{Z}^{d}} \sum_{i=0,1,2} H(x, i, \eta)\left[f\left(\eta^{x, i}\right)-f(\eta)\right]
$$

for any $\eta \in\{0,1,2\}^{\mathbb{Z}^{d}}$ and $f$ in the domain of $\Omega$, where

$$
\eta^{x, i}(y)= \begin{cases}\eta(y) & \text { if } y \neq x \\ i & \text { if } y=x\end{cases}
$$

and

$$
H(x, i, \eta)= \begin{cases}1 & \text { if } \eta(x)=2 \text { and } i=0, \\ 1+\delta & \text { if } \eta(x)=1 \text { and } i=0, \\ \gamma & \text { if } \eta(x)=1 \text { and } i=2, \\ \lambda \sum_{y: y \sim x} 1_{\{\eta(y)=2\}} & \text { if } \eta(x)=0 \text { and } i=1, \\ 0 & \text { else }\end{cases}
$$

for any $x \in \mathbb{Z}^{d}$ and $i \in\{0,1,2\}$, where $\lambda, \delta, \gamma$ are positive constants and $1_{A}$ is the indicator function of the event $A$, i.e., $1_{A}=1$ on the event $A$ while $1_{A}=0$ on the complementary set of $A$. Note that the domain of $\Omega$ is a dense subset of $C(X)$, the space of continuous functions on $X$.

Intuitively, the two-stage contact process describes the spread of an epidemic on the graph $\mathbb{Z}^{d}$. The vertices in state 0 are healthy and vertices in state 1 are semiinfected while vertices in state 2 are fully-infected. A fully-infected vertex waits for an exponential time with rate 1 to become healthy. A semi-infected vertex waits for an exponential time with rate $1+\delta$ to become healthy while it waits for an exponential time with rate $\gamma$ to become fully-infected, depending on which moment comes first. A healthy vertex is infected to become semi-infected at rate proportional to the number of fully-infected neighbors.

The two-stage contact process $\left\{\eta_{t}\right\}_{t \geq 0}$ is introduced in Krone (1999). In Krone (1999), a duality relationship between the two-stage contact process and a 'on-off' process is given. Several important open questions are proposed at the end of Krone (1999), some of which are answered in Foxall (2015) authored by Foxall. For instance, it is shown in Foxall (2015) that the complete convergence theorem holds for the two-stage contact process, i.e., the process converges weakly to a convex combination of two invariant distributions $\delta_{0}$ and $\nu$, where $\delta_{0}$ is concentrated on the state where all the vertices are in state 0 and $\nu$ is the upper invariant law of the process, the definition of which we will review in the next section.

Informally, the basic contact process introduced in Harris (1974) can be considered as a 'special two-stage contact process' with $\gamma=+\infty$, where a semi-infected vertex becomes fully-infected immediately such that the two states 1 and 2 can be combined as one infected state. For a detailed survey about the study of the basic contact process, see Liggett (1985, Chapter 9) and Liggett (1999, Part 1), two books authored by Liggett. Note that here and in several further places we are only giving an intuitive relation between the basic and two-stage contact processes when we talk about setting $\gamma=+\infty$. Our main results given in the next section hold rigorously for $\gamma \in(0,+\infty)$. 


\section{Main results}

In this section we give our main results. First we introduce some notation and definitions. For any $t \geq 0$, we define

$$
C_{t}=\left\{x \in \mathbb{Z}^{d}: \eta_{t}(x)=2\right\}
$$

as the set of fully-infected vertices at the moment $t$ and

$$
D_{t}=\left\{x \in \mathbb{Z}^{d}: \eta_{t}(x)=1\right\}
$$

as the set of semi-infected vertices at the moment $t$ and $I_{t}=C_{t} \cup D_{t}$ as the set of infected vertices at the moment $t$. For $C, D \subseteq \mathbb{Z}^{d}$, we write $\eta_{t}, C_{t}, D_{t}, I_{t}$ as $\eta_{t}^{(C, D)}, C_{t}^{(C, D)}, D_{t}^{(C, D)}, I_{t}^{(C, D)}$ when $C_{0}=C, D_{0}=D$. If $C=\{x\}($ resp. $D=\{x\})$ for some $x \in \mathbb{Z}^{d}$, we write $(C, D)$ as $(x, D)$ (resp. $\left.(C, x)\right)$ instead of $(\{x\}, D)$ (resp. $(C,\{x\}))$. Throughout this paper, we assume that $\delta, \gamma$ are fixed strictly positive constants. We use $P_{\lambda}$ to denote the probability measure of the two-stage contact process with infection rate $\lambda$. The expectation with respect to $P_{\lambda}$ is denoted by $E_{\lambda}$. We write $P_{\lambda}, E_{\lambda}$ as $P_{\lambda, d}, E_{\lambda, d}$ when we need to point out the dimension $d$ of the lattice.

It is shown in Krone (1999) that $P_{\lambda}\left(I_{t}^{(O, \emptyset)} \neq \emptyset\right.$ for all $\left.t \geq 0\right)$ is increasing with $\lambda$, then it is reasonable to define

$$
\lambda_{c}=\sup \left\{\lambda: P_{\lambda}\left(I_{t}^{(O, \emptyset)} \neq \emptyset \text { for all } t \geq 0\right)=0\right\} .
$$

$\lambda_{c}$ is called the critical value of the infection rate. When $\lambda<\lambda_{c}$, the infected vertices of the two-stage contact process with infection rate $\lambda$ die out with probability one conditioned on $O$ being the unique initially fully-infected vertex while other vertices being healthy at $t=0$.

It is shown in Krone (1999) that the two-stage contact process $\left\{\eta_{t}\right\}_{t \geq 0}$ is a monotonic process with respect to the partial order $\preceq$ on $\{0,1,2\}^{\mathbb{Z}^{d}}$ such that $\eta \preceq \xi$ when and only when $\eta(x) \leq \xi(x)$ for all $x \in \mathbb{Z}^{d}$. As a result, $\eta_{t}^{\left(\mathbb{Z}^{d}, \emptyset\right)}$ converges weakly to an invariant distribution $\nu$ as $t \rightarrow+\infty . \nu$ is called the upper invariant law of the two-stage contact process. We write $\nu$ as $\nu_{\lambda}$ when we need to point out the infection rate $\lambda$ and further write $\nu_{\lambda}$ as $\nu_{\lambda, d}$ when we need to point out the dimension $d$ of the lattice.

It is shown in Krone (1999) that $\nu_{\lambda}(\eta(O) \neq 0)$ is increasing with $\lambda$, so it is reasonable to define

$$
\widetilde{\lambda}_{c}=\sup \left\{\lambda: \nu_{\lambda}(\eta(O) \neq 0)=0\right\} .
$$

The following proposition is proved in Foxall (2015) by Foxall.

Proposition 2.1. (Foxall, 2015) If $\lambda_{c}$ and $\widetilde{\lambda}_{c}$ are defined as in Equations (2.1) and (2.2) respectively, then

$$
\lambda_{c}=\widetilde{\lambda}_{c}
$$

Proposition 2.1 shows that the above two types of critical values of the two-stage contact process are equal. So from now on, we use $\lambda_{c}$ to denote both of them.

We write $\lambda_{c}$ as $\lambda_{c}(d)$ when we need to point out the dimension $d$ of the lattice $\mathbb{Z}^{d}$. It is shown in Xue (2018) authored by Xue that

$$
\lim _{d \rightarrow+\infty} 2 d \lambda_{c}(d)=\frac{1+\delta+\gamma}{\gamma}
$$


As a result, for sufficiently large $d$ and $\lambda>\frac{1+\delta+\gamma}{\gamma}$,

$$
\nu_{\frac{\lambda}{2 d}, d}(\eta(O) \neq 0)>0 \text {. }
$$

One of our main results in this paper gives a more precise result than the above inequality. To give this result, we define

$$
\pi(A, B)=\nu(\eta(x) \neq 2 \text { for any } x \in A \text { and } \eta(y)=0 \text { for any } y \in B)
$$

for any $A, B \subseteq \mathbb{Z}^{d}$ such that $A \cap B=\emptyset$. We write $\pi(A, B)$ as $\pi(A, B, \lambda, d)$ when we need to point out the infection rate $\lambda$ and the dimension $d$ of the lattice. Then, for any $d \geq 1, m, n \geq 0$ and $\lambda>\frac{1+\delta+\gamma}{\gamma}$, we define

$$
\begin{aligned}
& \Pi(m, n, \lambda, d)= \\
& \sup \left\{\left|\pi\left(A, B, \frac{\lambda}{2 d}, d\right)-\left(1-\frac{\lambda \gamma-(1+\delta+\gamma)}{\lambda(\gamma+1)}\right)^{m}\left(\frac{1+\delta+\gamma}{\lambda \gamma}\right)^{n}\right|:\right. \\
& \left.A, B \subseteq \mathbb{Z}^{d},|A|=m,|B|=n, A \cap B=\emptyset\right\},
\end{aligned}
$$

where $|A|$ is the cardinality of $A$. Then, we obtain the following theorem, which is our first main result.

Theorem 2.2. For any $\lambda>\frac{1+\delta+\gamma}{\gamma}$ and integers $m, n \geq 0$,

$$
\lim _{d \rightarrow+\infty} \Pi(m, n, \lambda, d)=0 .
$$

Intuitively, Theorem 2.2 shows that $\nu_{\frac{\lambda}{2 d}, d}$ with $\lambda>\frac{1+\delta+\gamma}{\gamma}$ and large $d$ is approximate to a product measure $\mathfrak{m}$ on $\{0,1,2\}^{\mathbb{Z}^{d}}$ such that $\left\{\eta(x): x \in \mathbb{Z}^{d}\right\}$ are independent under $\mathfrak{m}$ and

$$
\begin{aligned}
& \mathfrak{m}(\eta(x)=0)=\frac{1+\delta+\gamma}{\lambda \gamma}, \mathfrak{m}(\eta(x)=2)=\frac{\lambda \gamma-(1+\delta+\gamma)}{\lambda(\gamma+1)} \\
& \text { while } \mathfrak{m}(\eta(x)=1)=\frac{\lambda \gamma-(1+\delta+\gamma)}{\lambda \gamma(\gamma+1)}
\end{aligned}
$$

for each $x \in \mathbb{Z}^{d}$.

As we have introduced, the basic contact process can be informally considered as the two-stage contact process with $\gamma=+\infty$. Let $\widetilde{\nu}$ be the upper invariant law of the basic contact process, then it is shown in Schonmann and Vares (1986) authored by Schonmann and Vares that

$$
\begin{aligned}
& \lim _{d \rightarrow+\infty} \sup \left\{\mid \widetilde{\nu}_{\frac{\lambda}{2 d}, d}(\eta(x)=0 \text { for all } x \in A)-\left(\frac{1}{\lambda}\right)^{m} \mid:\right. \\
&\left.A \subseteq \mathbb{Z}^{d},|A|=m\right\}=0
\end{aligned}
$$

for each $m \geq 0$ and $\lambda>1$. Since $\lim _{\gamma \rightarrow+\infty} \frac{1+\delta+\gamma}{\lambda \gamma}=\frac{1}{\lambda}$, Theorem 2.2 can be considered as an extension of Equation (2.3).

Theorem 2.2 is consistent with a non-rigorous mean-field analysis. For large $d$, $\left\{\eta_{t}(x)\right\}_{x \in \mathbb{Z}^{d}}$ are considered as approximately independent. Then, $P_{\frac{\lambda}{2 d}}\left(\eta_{t}(x)=1\right)$ 
and $P_{\frac{\lambda}{2 d}}\left(\eta_{t}(x)=2\right)$ approach to the solution $\left(x_{t}, y_{t}\right)$ to the ODE

$$
\left\{\begin{array}{l}
\frac{d}{d t} x_{t}=-(1+\delta+\gamma) x_{t}+\lambda\left(1-x_{t}-y_{t}\right) y_{t} \\
\frac{d}{d t} y_{t}=-y_{t}+\gamma x_{t}
\end{array}\right.
$$

which has strictly positive fixed points $x_{\infty}=\frac{\lambda \gamma-(1+\gamma+\delta)}{\lambda \gamma(\gamma+1)}$ and $y_{\infty}=\frac{\lambda \gamma-(1+\delta+\gamma)}{\lambda(\gamma+1)}$ when $\lambda>\frac{1+\delta+\gamma}{\gamma}$.

Our second main result is about the asymptotic behavior of $\lambda_{c}(d)$ as $d \rightarrow+\infty$. According to the approach introduced in Xue (2018),

$$
0 \leq 2 d \lambda_{c}(d)-\frac{1+\delta+\gamma}{\gamma} \leq O\left(\frac{(\log d)^{\frac{3}{\log d}}}{\log d}\right)
$$

as $d$ grows to infinity. The following theorem gives the stronger conclusion that $2 d \lambda_{c}(d)-\frac{1+\delta+\gamma}{\gamma}$ and $1 / d$ are infinitesimals of the same order as $d \rightarrow+\infty$, which is our second main result.

Theorem 2.3. If $\lambda_{c}$ is defined as in Equation (2.1), then

$$
f_{1} \leq \liminf _{d \rightarrow+\infty} d\left(2 d \lambda_{c}(d)-\frac{1+\delta+\gamma}{\gamma}\right) \leq \limsup _{d \rightarrow+\infty} d\left(2 d \lambda_{c}(d)-\frac{1+\delta+\gamma}{\gamma}\right) \leq f_{2}
$$

where

$$
f_{1}=\frac{1}{2}\left(1+\frac{1}{\gamma}\right) \frac{(1+\delta+\gamma)^{2}}{\gamma(2+\delta+\gamma)} \quad \text { and } \quad f_{2}=\frac{1+\gamma+\delta}{\gamma}\left(1+\frac{1}{\gamma}\right)
$$

which are constants only depend on $\gamma$ and $\delta$.

The counterpart of Theorem 2.3 for the critical value of the basic contact process is obtained in former references. Let $\beta_{c}(d)$ be the critical value of the basic contact process on $\mathbb{Z}^{d}$. In Holley and Liggett (1981) authored by Holley and Liggett, it is shown that

$$
\limsup _{d \rightarrow+\infty} d\left(2 d \beta_{c}(d)-1\right) \leq \frac{3}{2}
$$

Griffeath improves the above result in Griffeath (1983) by showing that

$$
\limsup _{d \rightarrow+\infty} d\left(2 d \beta_{c}(d)-1\right) \leq 1
$$

In Section 3.5 of Liggett (1985), it is shown that

$$
\beta_{c}(d) \geq \frac{1}{2 d-1}
$$

for all $d$ and hence $\liminf _{d \rightarrow+\infty} d\left(2 d \beta_{c}(d)-1\right) \geq \frac{1}{2}$. In conclusion, former references show that

$$
\frac{1}{2} \leq \liminf _{d \rightarrow+\infty} d\left(2 d \beta_{c}(d)-1\right) \leq \limsup _{d \rightarrow+\infty} d\left(2 d \beta_{c}(d)-1\right) \leq 1 .
$$

Note that $\lim _{\gamma \rightarrow+\infty} f_{1}(\gamma)=\frac{1}{2}$ while $\lim _{\gamma \rightarrow+\infty} f_{2}(\gamma)=1$, hence Theorem 2.3 can be considered as an extension of Equation (2.4).

It is natural to ask whether there exists $f_{3}$ such that

$$
\lim _{d \rightarrow+\infty} d\left(2 d \lambda_{c}(d)-\frac{1+\delta+\gamma}{\gamma}\right)=f_{3} .
$$

This question is open even for the basic contact process, i.e, the case where $\gamma=+\infty$. We will work on this question in follow-up work. 
The remainder of this paper is devoted to the proofs of Theorems 2.2 and 2.3. As we have discussed above, Theorem 2.3 is an improvement of the main result given in Xue (2018). To prove Theorem 2.3, we utilize different approaches with those introduced in Xue (2018) to bound $\lambda_{c}(d)$ from below and above. In Xue (2018), the lower bound $\frac{1+\delta+\gamma}{2 d \gamma}$ of $\lambda_{c}(d)$ is obtained through a coupling between the two-stage contact process and an auxiliary process with state space $\left\{\mathbb{Z}_{+}^{2}\right\}^{\mathbb{Z}^{d}}$, which is an analogue of the binary contact path process introduced in Griffeath (1983) by Griffeath. In Section 3 of this paper, we utilize a graphical representation of the two-stage contact process introduced in Krone (1999) by Krone and a strategy similar with that introduced in Section 3.5 of Liggett (1985) to show that

$$
\liminf _{d \rightarrow+\infty} d\left(2 d \lambda_{c}(d)-\frac{1+\delta+\gamma}{\gamma}\right) \geq f_{1} .
$$

In Xue (2018), the upper bound $\frac{1+\delta+\gamma}{2 d \gamma}+O\left(\frac{(\log d)^{\frac{3}{\log d}}}{2 d \log d}\right)$ of $\lambda_{c}(d)$ is obtained through an approach similar with that introduced in Kesten (1990) by Kesten to study critical probabilities of high dimensional site percolation models, which relates the estimation of the upper bound of the critical value of the model (percolation or contact process) with the times of collisions of two independent oriented random walks on $\mathbb{Z}^{d}$. To improve the above upper bound, in Section 4 of this paper, we introduce a process with state space $([0,+\infty) \times[0,+\infty))^{\mathbb{Z}^{d}}$, which is an analogue of the normalized binary contact path process introduced in Griffeath (1983) by Griffeath, and utilize a strategy similar with that introduced in Section 9.3 of Liggett (1985) to show that

$$
\limsup _{d \rightarrow+\infty} d\left(2 d \lambda_{c}(d)-\frac{1+\delta+\gamma}{\gamma}\right) \leq f_{2}
$$

As we have discussed above, Theorem 2.3 is an analogue of the main result given in Schonmann and Vares (1986) authored by Schonmann and Vares. Roughly speaking, the proof of the main result in Schonmann and Vares (1986) has two steps. First, according to a coupling of the basic contact process and a branching process, the probability that the number of infected vertices of a high-dimensional basic contact process reaches a large number $M$ for some $t>0$ is estimated. Second, according to a coupling of a high-dimensional basic contact process and a given low-dimensional one, it is proved that the survival probability of the basic contact process on $\mathbb{Z}^{d}$ with $M$ initially infected vertices and infection rate $\frac{\lambda}{2 d}$, where $\lambda>1$, converges to 1 as the dimension $d$ first grows to infinity and then the number $M$ grows to infinity. Lemma 9.14 of Harris (1976) authored by Harris is crucial for the second step, which shows that the survival probability of a given $l$-dimensional supercritical basic contact process converges to 1 as the number $M$ of initially infected vertices grows to infinity. Our proof of Theorem 2.2 given in Sections 5 and 6 has similar two steps. The duality relationship between the two-stage contact process and a so-called 'on-off' process introduced in Krone (1999) makes $1-\pi(A, B)$ the survival probability of types 1 and 2 vertices of the 'on-off' process. We first give a coupling of the 'on-off' process and a two-type branching process to estimate the probability that the number of types 1 and 2 vertices of the 'onoff' process reaches a large number $M$ for some $t>0$. We second show that the survival probability of types 1 and 2 vertices of the 'on-off' process on $\mathbb{Z}^{d}$ with $M$ initial types 1 and 2 vertices and parameter $\frac{\lambda}{2 d}$, where $\lambda>\frac{1+\gamma+\delta}{\gamma}$, converges 
to 1 as the dimension $d$ first grows to infinity and then the number $M$ grows to infinity. However, an analogue version of Lemma 9.14 of Harris (1976) for the 'onoff' process has not been proved yet. Hence in the second step we still resort to the strategy introduced in Section 9.3 of Liggett (1985). We utilize the coupling relationships between the three models, i.e., the two-stage contact process, the 'onoff' process and our auxiliary model with state space $([0,+\infty) \times[0,+\infty))^{\mathbb{Z}^{d}}$, to bound the survival probability of types 1 and 2 vertices of the 'on-off' process with $M$ initial types 1 and 2 vertices from below for large $M$. For details, see Sections 5 and 6.

\section{Proof of Equation (2.5)}

In this section we give the proof of Equation (2.5). Our proof follows the strategy introduced in Section 3.5 of (Liggett, 1985) to attest Equation (3.5.14) of the same book, which shows that the critical value of the basic contact process on $\mathbb{Z}^{d}$ is more than or equal to $\frac{1}{2 d-1}$. First we review the graphical representation of the two-stage contact process introduced in Krone (1999). According to this graphical representation, for given $A, B \subseteq \mathbb{Z}^{d}$ such that $A \cap B=\emptyset$, the family of processes

$$
\left\{\left\{I_{t}^{(C, D)}\right\}_{t \geq 0}: C \subseteq A, D \subseteq B\right\}
$$

can be coupled on the same probability space. We consider the set $\mathbb{Z}^{d} \times[0,+\infty)$, i.e, there is a time axis $[0,+\infty)$ on each vertex $x \in \mathbb{Z}^{d}$. For each $x \in \mathbb{Z}^{d}$, let $\left\{Y_{x}(t)\right\}_{t \geq 0}$ be a Poisson process with rate one, then we put a ' $\Delta$ ' on $(x, s)$ for each event moment $s$ of $Y_{x}(\cdot)$. For each $x \in \mathbb{Z}^{d}$, let $\left\{W_{x}(t)\right\}_{t \geq 0}$ be a Poisson process with rate $\delta$, then we put a '*' on $(x, r)$ for each event moment $r$ of $W_{x}(\cdot)$. For each $x \in \mathbb{Z}^{d}$, let $\left\{V_{x}(t)\right\}_{t \geq 0}$ be a Poisson process with rate $\gamma$, then we put a ' $\diamond$ ' on $(x, u)$ for each event moment $u$ of $V_{x}(\cdot)$. For any $x, y \in \mathbb{Z}^{d}$ such that $x \sim y$, let $\left\{U_{(x, y)}(t)\right\}_{t \geq 0}$ be a Poisson process with rate $\lambda$, then we put a ' $\rightarrow$ ' from $(x, v)$ to $(y, v)$ for each event moment $v$ of $U_{(x, y)}(\cdot)$. We assume that all these Poisson processes are independent. Note that we care about the order of $x$ and $y$, hence $U_{(x, y)} \neq U_{(y, x)}$.

Now assuming that $A, B \subseteq \mathbb{Z}^{d}$ such that $A \cap B=\emptyset$, then we put a ' $\diamond$ ' on $(x, 0)$ for each $x \in A$. For $x \in A \cup B, y \in \mathbb{Z}^{d}$ and $t>0$, we say that there is an infection path from $(x, 0)$ to $(y, t)$ when there exist $n \geq 0, x=x_{0} \sim x_{1} \sim x_{2} \sim \ldots \sim x_{n}=y$ and $0=t_{0}<t_{1}<t_{2}<\ldots<t_{n}<t_{n+1}=t$ such that the following five conditions all hold.

(1) There is an ' $\rightarrow$ ' from $\left(x_{i-1}, t_{i}\right)$ to $\left(x_{i}, t_{i}\right)$ for all $1 \leq i \leq n$.

(2) There exists at least one ' $\diamond$ ' on $\left\{x_{i}\right\} \times\left[t_{i}, t_{i+1}\right)$ for all $0 \leq i \leq n-1$.

(3) There is no ' $\Delta$ ' on $\left\{x_{i}\right\} \times\left[t_{i}, t_{i+1}\right)$ for all $0 \leq i \leq n$.

(4) For each $0 \leq i \leq n-1$, let

$$
m_{i}=\inf \left\{s \in\left[t_{i}, t_{i+1}\right): \text { there is a ' } \diamond \text { ' on }\left(x_{i}, s\right)\right\},
$$

then there is no ' $*$ ' on $\left\{x_{i}\right\} \times\left[t_{i}, m_{i}\right)$ for all $0 \leq i \leq n$.

(5) Let

$$
m_{n}=\inf \left\{s \in\left[t_{n}, t\right): \text { there is a ' } \diamond \text { ' on }\left(x_{i}, s\right)\right\},
$$

then there is no '*' on $\{y\} \times\left[t_{n}, m_{n}\right)$ if $m_{n}<+\infty$ while there is no '*' on $\{y\} \times\left[t_{n}, t\right)$ if $m_{n}=+\infty$.

Note that condition (2) ensures that $m_{i}<+\infty$ for $0 \leq i \leq n-1$ while $m_{n}$ may equals inf $\emptyset=+\infty$, so condition (5) contains two cases. 
For $C \subseteq A, D \subseteq B$ and $t \geq 0$, we define

$$
\begin{gathered}
\widehat{I}_{t}^{(C, D)}=\left\{y \in \mathbb{Z}^{d}: \text { there is an infection path from }(x, 0)\right. \\
\text { to }(y, t) \text { for some } x \in C \cup D\} .
\end{gathered}
$$

According to the theory of the graphical method introduced in Harris (1978) authored by Harris, it is easy to check that $\left\{\widehat{I}_{t}^{(C, D)}\right\}_{t \geq 0}$ and $\left\{I_{t}^{(C, D)}\right\}_{t \geq 0}$ are identically distributed, where

$$
I_{t}^{(C, D)}=\left\{x: \eta_{t}^{(C, D)}(x)>0\right\}
$$

defined as in Section 2. For readers not familiar with the graphical method, we give an intuitive explanation here. An (semi- of fully-) infected vertex $x$ becomes healthy at the event moment of $Y_{x}(\cdot)$. If $x$ is semi-infected, it also becomes healthy at the event moment of $W_{x}(\cdot)$ while becomes fully-infected at the event moment of $V_{x}(\cdot)$. If $x$ is fully infected while the neighbor $y$ of $x$ is healthy, then $y$ is infected by $x$ to become semi-infected when there is an ' $\rightarrow$ ' from $x$ to $y$. As a result, if there is an infection path from $(x, 0)$ to $(y, t)$ for $x \in C \cup D$, then for each $i \leq n-1$, $x_{i}$ is (semi- or fully-) infected at $t_{i}$ and is full-infected at $m_{i}$ while maintains fullyinfected till $t_{i+1}$ to ensure that $x_{i+1}$ is infected at $t_{i+1}$ for all $0 \leq i \leq n-1$. Hence, $y=x_{n}$ is infected at $t_{n}$. If $m_{n}<+\infty$, then $y$ becomes fully-infected at $m_{n}$ and maintains fully-infected till $t$. If $m_{n}=+\infty$, then $y$ maintains semi-infected till $t$. Therefore,

$$
\widehat{I}_{t}^{(C, D)} \subseteq\left\{y: \eta_{t}^{(C, D)}(y)>0\right\} .
$$

The opposite direction that $\widehat{I}_{t}^{(C, D)} \supseteq\left\{y: \eta_{t}^{(C, D)}(x)>0\right\}$ follows from similar analysis, we omit the details.

From now on, we assume that $\left\{\left\{I_{t}^{(C, D)}\right\}_{t \geq 0}: C \subseteq A, D \subseteq B\right\}$ are coupled on the same probability space such that

$$
\begin{gathered}
I_{t}^{(C, D)}=\left\{y \in \mathbb{Z}^{d}: \text { there is an infection path from }(x, 0)\right. \\
\text { to }(y, t) \text { for some } x \in C \cup D\}
\end{gathered}
$$

for any $t>0$. According to Equation (3.1), we have the following lemma, which is crucial for us to prove Equation (2.5).

Lemma 3.1. For $A, B \subseteq \mathbb{Z}^{d}$ such that $A \cap B=\emptyset$ and $C_{+}, C_{-} \subseteq A$ while $D_{+}, D_{-} \subseteq$ $B$,

$$
\begin{aligned}
& P_{\lambda}\left(I_{t}^{\left(C_{+} \cup C_{-}, D_{+} \cup D_{-}\right)} \neq \emptyset\right)+P_{\lambda}\left(I_{t}^{\left(C_{+} \cap C_{-}, D_{+} \cap D_{-}\right)} \neq \emptyset\right) \\
& \leq P_{\lambda}\left(I_{t}^{\left(C_{+}, D_{+}\right)} \neq \emptyset\right)+P_{\lambda}\left(I_{t}^{\left(C_{-}, D_{-}\right)} \neq \emptyset\right)
\end{aligned}
$$

for any $t \geq 0$.

Note that Lemma 3.1 is an analogue of Proposition 3.5.9 of Liggett (1985).

Proof of Lemma 3.1: For $C \subseteq A$ and $D \subseteq B$, we use $H_{t}(C, D)$ to denote the indicator function of the event that there exists an infection path from $(x, 0)$ to $(y, 0)$ for some $x \in C \cup D$ and $y \in \mathbb{Z}^{d}$. Then, we claim that

$$
\begin{aligned}
& H_{t}\left(C_{+} \cup C_{-}, D_{+} \cup D_{-}\right)+H_{t}\left(C_{+} \cap C_{-}, D_{+} \cap D_{-}\right) \\
& \leq H_{t}\left(C_{+}, D_{+}\right)+H_{t}\left(C_{-}, D_{-}\right)
\end{aligned}
$$

for $C_{+}, C_{-} \subseteq A$ and $D_{+}, D_{-} \subseteq B$. 
Lemma 3.1 follows from inequality (3.2) directly since

$$
P_{\lambda}\left(I_{t}^{(C, D)} \neq \emptyset\right)=E_{\lambda}\left(H_{t}(C, D)\right)
$$

according to Equation (3.1).

Now we prove inequality (3.2). For the case where $H_{t}\left(C_{+} \cup C_{-}, D_{+} \cup D_{-}\right)=$ $H_{t}\left(C_{+} \cap C_{-}, D_{+} \cap D_{-}\right)=0$, inequality (3.2) holds trivially. To check other cases, first note that $H\left(C_{1}, D_{1}\right) \leq H\left(C_{2}, D_{2}\right)$ when $C_{1} \subseteq C_{2}, D_{1} \subseteq D_{2}$ according to the definition of $H$. Consequently, for the case where $H_{t}\left(C_{+} \cap C_{-}, D_{+} \cap D_{-}\right)=1$, since $C_{+} \cap C_{-} \subseteq C_{+}, C_{-}, C_{+} \cup C_{+}$and $D_{+} \cap D_{-} \subseteq D_{+}, D_{-}, D_{+} \cup D_{-}$, we have

$$
H_{t}\left(C_{+} \cup C_{-}, D_{+} \cup D_{-}\right)=H_{t}\left(C_{+}, D_{+}\right)=H_{t}\left(C_{-}, D_{-}\right)=1
$$

and hence both sides of inequality (3.2) are equal to 2. For the case where $H_{t}\left(C_{+} \cap\right.$ $\left.C_{-}, D_{+} \cap D_{-}\right)=0$ while $H_{t}\left(C_{+} \cup C_{-}, D_{+} \cup D_{-}\right)=1$, there is an infection path from $(x, 0)$ to $(y, 0)$ for some $x \in C_{+} \cup C_{-} \cup D_{+} \cup D_{-}$and $y \in \mathbb{Z}^{d}$. Since such $x$ is in $C_{+} \cup D_{+}$or $C_{-} \cup D_{-}$, the right hand side of inequality (3.2) is more than or equal to 1 while the left hand side is 1 and hence inequality (3.2) holds. In conclusion, inequality (3.2) holds and the proof is complete.

For simplicity, we denote

$$
\begin{aligned}
& \alpha=P_{\lambda}\left(I_{t}^{(\emptyset, O)} \neq \emptyset \text { for all } t>0\right) \\
& q_{1}=P_{\lambda}\left(I_{t}^{(O, \emptyset)} \neq \emptyset \text { for all } t>0\right) \\
& k_{1}=P_{\lambda}\left(I_{t}^{\left(O, e_{1}\right)} \neq \emptyset \text { for all } t>0\right) \\
& k_{2}=\sup \left\{P_{\lambda}\left(I_{t}^{\left(O,\left\{e_{1}, y\right\}\right)} \neq \emptyset \text { for all } t>0\right): y \sim O, y \neq e_{1}\right\} \\
& q_{2}=P_{\lambda}\left(I_{t}^{\left(\left\{O, e_{1}\right\}, \emptyset\right)} \neq \emptyset \text { for all } t>0\right) \\
& q_{3}=\sup \left\{P_{\lambda}\left(I_{t}^{\left(\left\{O, e_{1}\right\}, y\right)} \neq \emptyset \text { for all } t>0\right): y \sim O, y \neq e_{1}\right\}
\end{aligned}
$$

where $e_{1}=(1,0, \ldots, 0)$ defined as in Section 1 , then we have the following lemma, which is an application of Lemma 3.1.

\section{Lemma 3.2.}

$$
k_{2} \leq 2 k_{1}-q_{1} \quad \text { and } \quad q_{3} \leq k_{1}+q_{2}-q_{1} .
$$

The motivation to establish Lemma 3.2 is that we want to estimate events involving three-dimensional marginals of the upper invariant law in terms of one- and two- dimensional marginals.

Proof of Lemma 3.2: For $y \sim O$ and $y \neq \emptyset$, let $C_{+}=C_{-}=A=\{O\}, B=\left\{e_{1}, y\right\}$, $D_{+}=\left\{e_{1}\right\}$ and $D_{-}=\{y\}$, then by Lemma 3.1 and the spatial homogeneity of the process,

$$
\begin{aligned}
& P_{\lambda}\left(I_{t}^{\left(O,\left\{e_{1}, y\right\}\right)} \neq \emptyset\right)+P_{\lambda}\left(I_{t}^{(O, \emptyset)} \neq \emptyset\right) \\
& \leq P_{\lambda}\left(I_{t}^{\left(O, e_{1}\right)} \neq \emptyset\right)+P_{\lambda}\left(I_{t}^{(O, y)} \neq \emptyset\right)=2 P_{\lambda}\left(I_{t}^{\left(O, e_{1}\right)} \neq \emptyset\right)
\end{aligned}
$$

for any $t \geq 0$. Let $t \rightarrow+\infty$ and then

$$
P_{\lambda}\left(I_{t}^{\left(O,\left\{e_{1}, y\right\}\right)} \neq \emptyset \text { for all } t>0\right)+q_{1} \leq 2 k_{1}
$$


$k_{2} \leq 2 k_{1}-q_{1}$ follows from which directly.

Let $C_{+}=A=\left\{O, e_{1}\right\}, C_{-}=\{O\}, B=\{y\}, D_{+}=\emptyset$ and $D_{-}=\{y\}$, then $q_{3} \leq k_{1}+q_{2}-q_{1}$ follows from the same analysis, we omit the details.

Now we give the proof of Equation (2.5).

Proof of Equation (2.5): Let $C_{0}=\left\{x: \eta_{0}(x)=2\right\}$ and $D_{0}=\left\{x: \eta_{0}(x)=1\right\}$ defined as in Section 2. If $C_{0}=\{O\}$ and $D_{0}=\emptyset$, then according to the property of independent exponential times, a neighbor of $O$ is infected to become semi-infected with probability $\frac{2 d \lambda}{1+2 d \lambda}$ while $O$ becomes healthy without infecting any neighbor with probability $\frac{1}{1+2 d \lambda}$. Therefore, according to the strong Markov property and the spatial homogeneity of the process,

$$
q_{1}=\frac{2 d \lambda}{2 d \lambda+1} k_{1}
$$

If $C_{0}=\emptyset$ and $D_{0}=\{O\}$, then according to a similar analysis,

$$
\alpha=\frac{\gamma}{1+\delta+\gamma} q_{1}
$$

If $C_{0}=\{O\}$ and $D_{0}=\left\{e_{1}\right\}$, then $\left(C_{0}, D_{0}\right)$ jumps to $(C, D)$ with probability

$$
\begin{cases}\frac{1+\delta}{(2 d-1) \lambda+2+\delta+\gamma} & \text { if } C=\{O\}, D=\emptyset \\ \frac{1}{(2 d-1) \lambda+2+\delta+\gamma} & \text { if } C=\emptyset, D=\left\{e_{1}\right\} \\ \frac{\gamma}{(2 d-1) \lambda+2+\delta+\gamma} & \text { if } C=\left\{O, e_{1}\right\}, D=\emptyset \\ \frac{\lambda}{(2 d-1) \lambda+2+\delta+\gamma} & \text { if } y \sim O, y \neq e_{1}, C=\{O\}, D=\left\{e_{1}, y\right\} \\ 0 & \text { else. }\end{cases}
$$

Then, according to the strong Markov property, spatial homogeneity of the process and Lemma 3.2,

$$
\begin{aligned}
k_{1} \leq & \frac{1+\delta}{(2 d-1) \lambda+2+\delta+\gamma} q_{1}+\frac{1}{(2 d-1) \lambda+2+\delta+\gamma} \alpha \\
& +\frac{\gamma}{(2 d-1) \lambda+2+\delta+\gamma} q_{2}+\frac{(2 d-1) \lambda}{(2 d-1) \lambda+2+\delta+\gamma}\left(2 k_{1}-q_{1}\right) .
\end{aligned}
$$

If $C_{0}=\left\{O, e_{1}\right\}$ and $D_{0}=\emptyset$, then according to Lemma 3.2 and a similar analysis with that leads to Equation (3.5),

$$
q_{2} \leq \frac{2}{2(2 d-1) \lambda+2} q_{1}+\frac{2(2 d-1) \lambda}{2(2 d-1) \lambda+2}\left(k_{1}+q_{2}-q_{1}\right)
$$

and hence

$$
q_{2} \leq \frac{(4 d-1) \lambda}{2 d \lambda+1} k_{1}
$$

By Equations (3.3), (3.4), (3.5) and (3.6),

$$
k_{1}(M(\lambda, \delta, \gamma)-1) \geq 0,
$$


where

$$
\begin{aligned}
& M(\lambda, \delta, \gamma)= \\
& \frac{\gamma}{(2 d-1) \lambda+2+\delta+\gamma} \frac{(4 d-1) \lambda}{2 d \lambda+1}+\frac{1}{(2 d-1) \lambda+2+\delta+\gamma} \frac{\gamma}{1+\delta+\gamma} \frac{2 d \lambda}{2 d \lambda+1} \\
& +\frac{1+\delta}{(2 d-1) \lambda+2+\delta+\gamma} \frac{2 d \lambda}{2 d \lambda+1}+\frac{(2 d-1) \lambda}{(2 d-1) \lambda+2+\delta+\gamma} \frac{2 d \lambda+2}{2 d \lambda+1} .
\end{aligned}
$$

By direct calculation, it is easy to check that

$$
M(\lambda, \delta, \gamma)<1
$$

when

$$
\lambda<\frac{1+\delta+\gamma}{2 d \gamma} \frac{2+\delta+\gamma}{1+\left[1-\frac{1}{2 d}\left(1+\frac{1}{\gamma}\right)\right](1+\delta+\gamma)} .
$$

Note that here we assume that $d$ is sufficiently large such that

$$
1+\left[1-\frac{1}{2 d}\left(1+\frac{1}{\gamma}\right)\right](1+\delta+\gamma)>0
$$

Then, by Equations (3.7) and (3.3), $k_{1}=0$ and hence $q_{1}=0$ when

$$
\lambda<\frac{1+\delta+\gamma}{2 d \gamma} \frac{2+\delta+\gamma}{1+\left[1-\frac{1}{2 d}\left(1+\frac{1}{\gamma}\right)\right](1+\delta+\gamma)} .
$$

Then, according to the definition of $\lambda_{c}(d)$ given in Equation (2.1),

$$
\lambda_{c}(d) \geq \frac{1+\delta+\gamma}{2 d \gamma} \frac{2+\delta+\gamma}{1+\left[1-\frac{1}{2 d}\left(1+\frac{1}{\gamma}\right)\right](1+\delta+\gamma)}
$$

Since

$$
\frac{2+\delta+\gamma}{1+\left[1-\frac{1}{2 d}\left(1+\frac{1}{\gamma}\right)\right](1+\delta+\gamma)}=1+\frac{1}{2 d}\left(1+\frac{1}{\gamma}\right) \frac{1+\delta+\gamma}{2+\delta+\gamma}+O\left(\frac{1}{d^{2}}\right)
$$

Equation (2.5) follows from Equation (3.8) directly.

\section{Proof of Equation (2.6)}

In this section we give the proof of Equation (2.6). Our proof is inspired by the strategy introduced in Section 9.3 of Liggett (1985). The outline of the proof is as follows. First we introduce a Markov-process $\left\{\rho_{t}=\left(\zeta_{t}, g_{t}\right): t \geq 0\right\}$ such that $\zeta_{t}, g_{t} \in[0,+\infty)^{\mathbb{Z}^{d}}$ and

$$
\eta_{t}(x)=2 \times 1_{\left\{\zeta_{t}(x)>0\right\}}+1_{\left\{\zeta_{t}(x)=0, g_{t}(x)>0\right\}} .
$$

We will choose proper transition rates function of $\rho_{t}$ such that

$$
E \zeta_{t}(x)=E g_{t}(x) \equiv 1
$$

Then, according to the Cauchy-Schwartz inequality,

$$
\liminf _{t \rightarrow+\infty} P\left(\eta_{t}(x)>0\right) \geq \frac{1}{\sup _{t \geq 0} E\left(\zeta_{t}^{2}(x)\right)} .
$$

The auxiliary process $\left\{\rho_{t}\right\}_{t \geq 0}$ belongs to the family of processes called linear systems introduced in Section 9 of Liggett (1985). By utilizing Theorem 9.3.1 of Liggett 
(1985), we show that $\left\{E\left(\zeta_{t}(x) \zeta_{t}(y)\right): \quad x, y \in \mathbb{Z}^{d}\right\}$ are given by some marginal dimensions of the solution to a linear ODE

$$
\frac{d}{d t} F_{t}=G_{\lambda} F_{t}
$$

where $F_{t}$ is a function from $X_{4}=\mathbb{Z}^{d} \times\{1,2,3\}$ to $\mathbb{R}$ and $G_{\lambda}$ is a $X_{4} \times X_{4}$ matrix. According to a lemma which is an analogue of Theorem 9.3.12 of Liggett (1985), we can show that $\sup _{t \geq 0} E\left(\zeta_{t}^{2}(x)\right)<+\infty$ if $G_{\lambda}$ has a positive eigenvector with respect to eigenvalue 0 . At last we find $\lambda$ which makes $G_{\lambda}$ have the aforesaid eigenvector and then such $\lambda$ is an upper bound of $\lambda_{c}$.

Now we give the details of the proof. As we have discussed above, first we define a continuous-time Markov process $\left\{\rho_{t}\right\}_{t \geq 0}$ as an auxiliary process for the proof.

The state space of $\left\{\rho_{t}\right\}_{t \geq 0}$ is $X_{2}=([0,+\infty) \times[0,+\infty))^{\mathbb{Z}^{d}}$, i.e., at each vertex $x \in \mathbb{Z}^{d}$ there is a spin $\rho(x)=(\zeta(x), g(x))$ such that $\zeta(x), g(x) \geq 0$. The generator of $\left\{\rho_{t}\right\}_{t \geq 0}$ is given by

$$
\begin{aligned}
\Omega_{2} f(\rho)= & \sum_{x \in \mathbb{Z}^{d}}\left[f\left(\rho^{x}\right)-f(\rho)\right]+\delta \sum_{x \in \mathbb{Z}^{d}}\left[f\left(\rho^{x,+}\right)-f(\rho)\right] \\
& +\gamma \sum_{x \in \mathbb{Z}^{d}}\left[f\left(\rho^{x,-}\right)-f(\rho)\right]+\lambda \sum_{x \in \mathbb{Z}^{d}} \sum_{y \sim x}\left[f\left(\rho^{x, y}\right)-f(\rho)\right]
\end{aligned}
$$

for any $\rho \in X_{2}$ and $f \in C\left(X_{2}\right)$, where

$$
\begin{gathered}
\rho^{x}(y)= \begin{cases}\rho(y)=(\zeta(y), g(y)) & \text { if } y \neq x, \\
(0,0) & \text { if } y=x,\end{cases} \\
\rho^{x,+}(y)= \begin{cases}\rho(y)=(\zeta(y), g(y)) & \text { if } y \neq x, \\
(\zeta(x), 0) & \text { if } y=x,\end{cases} \\
\rho^{x,-}(y)= \begin{cases}\rho(y)=(\zeta(y), g(y)) & \text { if } y \neq x, \\
\left(\zeta(x)+\frac{1}{\gamma} g(x), 0\right) & \text { if } y=x,\end{cases}
\end{gathered}
$$

and

$$
\rho^{x, y}(z)= \begin{cases}\rho(z)=(\zeta(z), g(z)) & \text { if } z \neq x \\ (\zeta(x), g(x)+b \zeta(y)) & \text { if } z=x\end{cases}
$$

where $b=\frac{1+\delta+\gamma}{2 d \lambda}$.

Here we choose the weights $\frac{1}{\gamma}$ and $b=\frac{1+\delta+\gamma}{2 d \lambda}$ for a technical reason. We will show later that these two weights can make the expectations of $\zeta_{t}(x)$ and $g_{t}(x)$ constant conditioned on $\zeta_{0}(x)=g_{0}(x)=1$ for each $x \in \mathbb{Z}^{d}$.

According to the generator $\Omega_{2}$ of $\left\{\rho_{t}\right\}_{t \geq 0}$, if the state of $\left\{\rho_{t}\right\}_{t \geq 0}$ jumps at a moment $s$, then $\zeta_{s}(x), g_{s}(x)$ are linear combinations of $\left\{\zeta_{s-}(y)\right\}_{y \in \mathbb{Z}^{d}}$ and $\left\{g_{s-}(y)\right\}_{y \in \mathbb{Z}^{d}}$ for each $x \in \mathbb{Z}^{d}$. As a result, $\left\{\rho_{t}\right\}_{t \geq 0}$ can be considered as a linear system, the theory of which is introduced in Chapter 9 of Liggett (1985).

In Chapter 9 of Liggett (1985), the state space of a linear system is defined to be $[0,+\infty)^{S}$, where $S$ is a countable set. Note that $\left\{\rho_{t}\right\}_{t \geq 0}$ is consistent with this definition since $([0,+\infty) \times[0,+\infty))^{\mathbb{Z}^{d}}$ can be identified with $[0,+\infty)^{\mathbb{Z}^{d} \times\{1,2\}}$ while $\mathbb{Z}^{d} \times\{1,2\}$ is a countable set.

The intuitive explanation of the evolution of $\left\{\rho_{t}\right\}_{t \geq 0}$ is as follows. For any $x \in \mathbb{Z}^{d}$, its state $\rho(x)=(\zeta(x), g(x))$ flips to $(0,0)$ at rate 1 , to $(\zeta(x), 0)$ at rate $\delta$, 
to $\left(\zeta(x)+\frac{1}{\gamma} g(x), 0\right)$ at rate $\gamma$ or to $(\zeta(x), g(x)+b \zeta(y))$ at rate $\lambda$ for each neighbor $y$.

From now on, we assume that $\rho_{0}(x)=(1,1)$ for any $x \in \mathbb{Z}^{d}$, then we define

$$
\phi_{t}(x)= \begin{cases}2 & \text { if } \zeta_{t}(x)>0, \\ 1 & \text { if } \zeta_{t}(x)=0 \text { and } g_{t}(x)>0, \\ 0 & \text { if } \zeta_{t}(x)=g_{t}(x)=0\end{cases}
$$

for any $t \geq 0$ and $x \in \mathbb{Z}^{d}$, where $\rho_{t}(x)=\left(\zeta_{t}(x), g_{t}(x)\right)$. The following lemma gives the relationship between $\left\{\rho_{t}\right\}_{t \geq 0}$ and the two-stage contact process.

Lemma 4.1. $\left\{\phi_{t}\right\}_{t \geq 0}$ is a version of the two-stage contact process with generator (1.1).

Proof: We only need to check that $\left\{\phi_{t}\right\}_{t \geq 0}$ evolves in the same way as that of the two-stage contact process $\left\{\eta_{t}\right\}_{t \geq 0}$. For any $x \in \mathbb{Z}^{d}$, if $\phi(x)=0$, i.e., $\rho(x)=(0,0)$, then $\phi(x)$ flips to 1 when and only when $\rho(x)$ flips to

$$
(0,0+b \zeta(y))=(0, b \zeta(y))
$$

for some $y \sim x$ such that $\zeta(y)>0$, i.e, $\phi(y)=2$. Since $\rho(x)$ flips to $(\zeta(x), g(x)+$ $b \zeta(y))$ at rate $\lambda, \phi(x)$ flips from 0 to 1 at rate

$$
\lambda \sum_{y \sim x} 1_{\{\phi(y)=2\}}=H(x, 1, \phi)
$$

defined in Equation (1.1). Through a similar way, it is easy to check that in every case $\phi(x)$ flips to a different state $i$ at rate $H(x, i, \phi)$ defined in Equation (1.1) and the proof is complete.

By Lemma 4.1, from now on we assume that $\left\{\rho_{t}\right\}_{t \geq 0}$ and the two-stage contact process $\left\{\eta_{t}\right\}_{t \geq 0}$ are defined under the same probability space such that

$$
\eta_{t}(x)= \begin{cases}2 & \text { if } \zeta_{t}(x)>0 \\ 1 & \text { if } \zeta_{t}(x)=0 \text { and } g_{t}(x)>0 \\ 0 & \text { if } \zeta_{t}(x)=g_{t}(x)=0\end{cases}
$$

for any $t \geq 0$ and $x \in \mathbb{Z}^{d}$. As a result,

$$
\nu_{\lambda}(\eta(O)=2)=\lim _{t \rightarrow+\infty} P_{\lambda}\left(\eta_{t}^{\left(\mathbb{Z}^{d}, \emptyset\right)}(O)=2\right)=\lim _{t \rightarrow+\infty} P_{\lambda}\left(\zeta_{t}(O)>0\right) .
$$

In view of Lemma 4.1, we have the following lemma, which gives an upper bound on the critical value $\lambda_{c}$.

Lemma 4.2. If $\lambda$ satisfies

$$
\sup _{t \geq 0} E_{\lambda}\left(\zeta_{t}^{2}(O)\right)<+\infty
$$

then $\lambda_{c} \leq \lambda$.

Proof: By Equation (4.2) and the Cauchy-Schwartz inequality,

$$
\begin{aligned}
\lim _{t \rightarrow+\infty} P_{\lambda}\left(\eta_{t}^{\left(\mathbb{Z}^{d}, \emptyset\right)}(O)=2\right) & =\lim _{t \rightarrow+\infty} P_{\lambda}\left(\zeta_{t}(O)>0\right) \\
& \geq \limsup _{t \rightarrow+\infty} \frac{\left(E_{\lambda} \zeta_{t}(O)\right)^{2}}{E_{\lambda}\left(\zeta_{t}^{2}(O)\right)} .
\end{aligned}
$$


For any given $x \in \mathbb{Z}^{d}$, we define

$$
f_{x}^{(1)}(\rho)=\zeta(x) \quad \text { and } \quad f_{x}^{(2)}(\rho)=g(x)
$$

for any $\rho=\left\{(\zeta(u), g(u)): u \in \mathbb{Z}^{d}\right\} \in X_{2}$, i.e., $f_{x}^{(i)}(\rho)$ is the $i$ th coordinate of $\rho(x)$. Let $\{S(t)\}_{t \geq 0}$ be the semi-group of $\left\{\rho_{t}\right\}_{t \geq 0}$. According to Theorem 9.1.27 of Liggett (1985), which is an extension version of the Hille-Yosida Theorem for the linear system, we can execute the calculation

$$
\frac{d}{d t} S(t) f_{x}^{(i)}(\rho)=S(t) \Omega_{2} f_{x}^{(i)}(\rho)
$$

for $i=1,2$. Then, taking $x=O$, according to the definition of $\Omega_{2}$,

$$
\left\{\begin{aligned}
\frac{d}{d t} E_{\lambda} \zeta_{t}(O) & =-E_{\lambda} \zeta_{t}(O)+\gamma E_{\lambda}\left(\frac{1}{\gamma} g_{t}(O)\right), \\
\frac{d}{d t} E_{\lambda} g_{t}(O) & =-(1+\delta+\gamma) E_{\lambda} g_{t}(O)+\lambda b \sum_{y: y \sim O} E_{\lambda} \zeta_{t}(y)
\end{aligned}\right.
$$

and hence

$$
\left\{\begin{aligned}
\frac{d}{d t} E_{\lambda} \zeta_{t}(O) & =-E_{\lambda} \zeta_{t}(O)+E_{\lambda} g_{t}(O) \\
\frac{d}{d t} E_{\lambda} g_{t}(O) & =-(1+\delta+\gamma) E_{\lambda} g_{t}(O)+(1+\delta+\gamma) E_{\lambda} \zeta_{t}(O)
\end{aligned}\right.
$$

by the spatial homogeneity of $\left\{\rho_{t}\right\}_{t \geq 0}$ and the initial condition where $\rho_{0}(x)=(1,1)$ for all $x \in \mathbb{Z}^{d}$.

Since $\zeta_{0}(O)=g_{0}(O)=1$, it is easy to check that the unique solution to ODE (4.4) is

$$
E_{\lambda} \zeta_{t}(O)=E_{\lambda} g_{t}(O) \equiv 1
$$

Then, by Equation (4.3),

$$
\begin{aligned}
\nu_{\lambda}(\eta(O)=2) & =\lim _{t \rightarrow+\infty} P_{\lambda}\left(\eta_{t}^{\left(\mathbb{Z}^{d}, \emptyset\right)}(O)=2\right) \\
& \geq \limsup _{t \rightarrow+\infty} \frac{1}{E_{\lambda}\left(\zeta_{t}^{2}(O)\right)} \geq \frac{1}{\sup _{t \geq 0} E_{\lambda}\left(\zeta_{t}^{2}(O)\right)}>0
\end{aligned}
$$

if $\lambda$ satisfies

$$
\sup _{t \geq 0} E_{\lambda}\left(\zeta_{t}^{2}(O)\right)<+\infty .
$$

Lemma 4.2 follows directly from Equation (4.5) and the equivalent definition of $\lambda_{c}$ given in Equation (2.2).

In view of Lemma 4.2, we want to bound $E_{\lambda}\left(\zeta_{t}^{2}(O)\right)$ from above. For this purpose, we define

$$
F_{t}(x, 1)=E_{\lambda}\left(\zeta_{t}(O) \zeta_{t}(x)\right), \quad F_{t}(x, 2)=E_{\lambda}\left(\zeta_{t}(O) g_{t}(x)\right)
$$

and $F_{t}(x, 3)=E_{\lambda}\left(g_{t}(O) g_{t}(x)\right)$ for each $x \in \mathbb{Z}^{d}$ and any $t \geq 0$. For any $t>0$, we define

$$
F_{t}=\left\{F_{t}(x, i): x \in \mathbb{Z}^{d}, i \in\{1,2,3\}\right\}
$$

as a function from $X_{4}=\mathbb{Z}^{d} \times\{1,2,3\}$ to $[0,+\infty)$. For a $X_{4} \times X_{4}$ matrix

$$
G=\{G((x, i),(y, j))\}_{(x, i),(y, j) \in X_{4}}
$$

and two functions $F_{+}, F_{-}$from $X_{4}$ to $\mathbb{R}$, we write

$$
F_{+}=G F_{-}
$$


if and only if

$$
F_{+}(x, i)=\sum_{(y, j) \in X_{4}} G((x, i),(y, j)) F_{-}(y, j)
$$

for any $(x, i) \in X_{4}$, as the product of finite dimensional matrixes. Then we have the following lemma.

Lemma 4.3. Let

$$
\frac{d}{d t} F_{t}=\left\{\frac{d}{d t} F_{t}(x, i):(x, i) \in X_{4}\right\}
$$

then

$$
\frac{d}{d t} F_{t}=G_{\lambda} F_{t}
$$

where $G_{\lambda}$ is a $X_{4} \times X_{4}$ matrix such that

$$
G_{\lambda}((x, i),(y, j))= \begin{cases}-2 & \text { if } x \neq O, i=1 \text { and }(y, j)=(x, 1), \\ 2 & \text { if } x \neq O, i=1 \text { and }(y, j)=(x, 2), \\ -1 & \text { if } x=O, i=1 \text { and }(y, j)=(O, 1), \\ 2 & \text { if } x=O, i=1 \text { and }(y, j)=(O, 2), \\ \frac{1}{\gamma} & \text { if } x=O, i=1 \text { and }(y, j)=(O, 3), \\ -(2+\delta+\gamma) & \text { if } x \neq O, i=2 \text { and }(y, j)=(x, 2), \\ 1 & \text { if } x \neq O, i=2 \text { and }(y, j)=(x, 3), \\ \frac{1+\delta+\gamma}{2 d} & \text { if } x \neq O, i=2, y \sim x \text { and } j=1, \\ -(1+\delta+\gamma) & \text { if } x=O, i=2 \text { and }(y, j)=(O, 2), \\ 1+\delta+\gamma & \text { if } x=O, i=2 \text { and }(y, j)=\left(e_{1}, 1\right), \\ -2(1+\delta+\gamma) & \text { if } x \neq O, i=3 \text { and }(y, j)=(x, 3), \\ \frac{1+\delta+\gamma}{d} & \text { if } x \neq O, i=3, y \sim x \text { and } j=2, \\ -(1+\delta+\gamma) & \text { if } x=O, i=3 \text { and }(y, j)=(O, 3), \\ 2(1+\delta+\gamma) & \text { if } x=O, i=3 \text { and }(y, j)=\left(e_{1}, 2\right), \\ \frac{(1+\delta+\gamma)^{2}}{2 d \lambda} & \text { if } x=O, i=3 \text { and }(y, j)=(O, 1), \\ 0 & \text { else. }\end{cases}
$$

Proof: According to the spatial homogeneity of the process $\left\{\rho_{t}\right\}_{t \geq 0}$,

$$
\begin{aligned}
& E_{\lambda}\left(\zeta_{t}(u) \zeta_{t}(v)\right)=F_{t}(u-v, 1)=F_{t}(v-u, 1), \\
& E_{\lambda}\left(\zeta_{t}(u) g_{t}(v)\right)=E_{\lambda}\left(\zeta_{t}(v) g_{t}(u)\right)=F_{t}(u-v, 2)=F_{t}(v-u, 2), \\
& E_{\lambda}\left(g_{t}(u) g_{t}(v)\right)=F_{t}(u-v, 3)=F_{t}(v-u, 3), \\
& F_{t}\left(e_{1}, i\right)=F_{t}(y, i)
\end{aligned}
$$

for any $y \sim O, u, v \in \mathbb{Z}^{d}$ and $i \in\{1,2,3\}$.

For given $x, y \in \mathbb{Z}^{d}$ and any $\rho=\left\{(\zeta(u), g(u)): u \in \mathbb{Z}^{d}\right\} \in X_{2}$, we define

$$
J_{x, y}^{(1)}(\rho)=\zeta(x) \zeta(y), J_{x, y}^{(2)}(\rho)=\zeta(x) g(y) \text { and } J_{x, y}^{(3)}(\rho)=g(x) g(y) .
$$

Theorem 9.3.1 of Liggett (1985) is an extended version of the Hille-Yosida Theorem for the linear system, according to which we can execute the calculation that

$$
\frac{d}{d t} S(t) J_{x, y}^{(i)}(\rho)=S(t) \Omega_{2} J_{x, y}^{(i)}(\rho)
$$


for any $x, y \in \mathbb{Z}^{d}$ and $i=1,2,3$. Therefore, by Equation (4.7) and the definition of $\Omega_{2}$,

$$
\begin{aligned}
& \frac{d}{d t} F_{t}(x, 1)=-2 F_{t}(x, 1)+2 F_{t}(x, 2) \\
& \frac{d}{d t} F_{t}(x, 2)=-(2+\delta+\gamma) F_{t}(x, 2)+F_{t}(x, 3)+\frac{1+\delta+\gamma}{2 d} \sum_{y: y \sim x} F_{t}(y, 1), \\
& \frac{d}{d t} F_{t}(x, 3)=-2(1+\delta+\gamma) F_{t}(x, 3)+\frac{(1+\delta+\gamma)}{d} \sum_{y: y \sim x} F_{t}(y, 2)
\end{aligned}
$$

when $x \neq O$ and

$$
\begin{aligned}
\frac{d}{d t} F_{t}(O, 1)= & -F_{t}(O, 1)+2 F_{t}(O, 2)+\frac{1}{\gamma} F_{t}(O, 3), \\
\frac{d}{d t} F_{t}(O, 2)= & -(1+\delta+\gamma) F_{t}(O, 2)+(1+\delta+\gamma) F_{t}\left(e_{1}, 1\right), \\
\frac{d}{d t} F_{t}(O, 3)= & -(1+\delta+\gamma) F_{t}(O, 3) \\
& +2(1+\delta+\gamma) F_{t}\left(e_{1}, 2\right)+\frac{(1+\delta+\gamma)^{2}}{2 d \lambda} F_{t}(O, 1) .
\end{aligned}
$$

Lemma 4.3 follows from Equations (4.8) and (4.9) directly.

According to Lemma 4.3, we have the following lemma about a sufficient condition for $\sup _{t \geq 0} E_{\lambda}\left(\zeta_{t}^{2}(O)\right)<+\infty$.

Lemma 4.4. If $\lambda$ satisfies that there exists $K_{\lambda}: X_{4} \rightarrow[0,+\infty)$ such that

$$
G_{\lambda} K_{\lambda}=0 \text { (zero function) }
$$

and $\inf _{(x, i) \in X_{4}} K_{\lambda}(x, i)>0$, then

$$
\sup _{t \geq 0} E_{\lambda}\left(\zeta_{t}^{2}(O)\right)<+\infty .
$$

Lemma 4.4 is an analogue of Theorem 9.3.12 of Liggett (1985). Readers familiar with the theory of linear systems can skip the following proof of this lemma.

To prove Lemma 4.4, we need to define the product of two $X_{4} \times X_{4}$ matrixes. For two $X_{4} \times X_{4}$ matrixes $G_{+}$and $G_{-}, G_{+} G_{-}$is defined as a $X_{4} \times X_{4}$ matrixes such that

$$
\left(G_{+} G_{-}\right)((x, i),(y, j))=\sum_{(u, l) \in X_{4}} G_{+}((x, i),(u, l)) G_{-}((u, l),(y, j))
$$

for any $(x, i),(y, j) \in X_{4}$, conditioned on the sum is absolute convergence (otherwise $G_{+} G_{-}$does not exists). Note that this definition is the same as that of the product of two finite dimensional matrix, except that the sum must convergence since there are infinite many terms. Then, we use $G_{\lambda}^{2}$ to denote $G_{\lambda} G_{\lambda}$ and define $G_{\lambda}^{n+1}=G_{\lambda}^{n} G_{\lambda}$ for $n \geq 2$ by induction. According to the definition of $G_{\lambda}$, for each $(x, i)$, the number of $(y, j)$ s satisfying

$$
G_{\lambda}((x, i),(y, j)) \neq 0
$$


is at most $3(2 d+1)$. Hence, the definition of $G_{\lambda}^{n}$ is reasonable for each $n \geq 2$ and $G_{\lambda}^{n}$ grows at most exponentially in $n$. Therefore,

$$
\sum_{n=0}^{+\infty} \frac{t^{n}\left|G_{\lambda}^{n}((x, i),(y, j))\right|}{n !}<+\infty
$$

for any $t \geq 0$ and $(x, i),(y, j) \in X_{4}$, where $G_{\lambda}^{1}=G_{\lambda}$ and $G_{\lambda}^{0}$ is the identity matrix. Consequently, it is reasonable to define $e^{t G_{\lambda}}$ as the $X_{4} \times X_{4}$ matrix such that

$$
e^{t G_{\lambda}}((x, i),(y, j))=\sum_{n=0}^{+\infty} \frac{t^{n} G_{\lambda}^{n}((x, i),(y, j))}{n !}
$$

for any $(x, i),(y, j) \in X_{4}$. The above way to define $e^{t G_{\lambda}}$ is surveyed in Liggett's book. For details, see page 114 of Liggett (1985). Now we can give the proof of Lemma 4.4.

Proof of Lemma 4.4: Since $G_{\lambda} K_{\lambda}=0, K_{\lambda}$ can be considered as the eigenvector of $G_{\lambda}$ with respect to the eigenvalue 0 , then according to a similar analysis with that in the theory of finite-dimensional linear algebra, $K_{\lambda}$ is the eigenvector of $e^{t G_{\lambda}}$ with respect to the eigenvalue $e^{t \times 0}=1$, i.e.,

$$
K_{\lambda}(x, i)=\sum_{(y, j) \in X_{4}} e^{t G_{\lambda}}((x, i),(y, j)) K_{\lambda}(y, j)
$$

for any $t \geq 0$ and $(x, i) \in X_{4}$.

For any function $K$ from $X_{4}$ to $\mathbb{R}$, we define

$$
\|K\|_{\infty}=\sup \left\{|K(x, i)|:(x, i) \in X_{4}\right\}
$$

as the $l_{\infty}$ norm of $K$. Then, we define $X_{5}$ as the set of functions from $X_{4}$ to $\mathbb{R}$ with finite $l_{\infty}$ norm $\|\cdot\|_{\infty}$. It is easy to check that there exists a constant $Q(\lambda)>0$ such that

$$
\left\|G_{\lambda} K_{+}-G_{\lambda} K_{-}\right\|_{\infty} \leq Q(\lambda)\left\|K_{+}-K_{-}\right\|_{\infty}
$$

for any $K_{-}, K_{+} \in X_{5}$, i.e., ODE (4.6) satisfies the Lipschitz condition. Since $X_{5}$ is a Banach space with the norm $\|\cdot\|_{\infty}$ and ODE (4.6) satisfies the Lipschitz condition, according to the theory of infinite dimensional order differential equations introduced in Chapter 19 of Lang (1983), the unique solution on $X_{5}$ to ODE (4.6) is

$$
F_{t}=e^{t G_{\lambda}} F_{0}
$$

i.e.,

$$
F_{t}(x, i)=\sum_{(y, j) \in X_{4}} e^{t G_{\lambda}}((x, i),(y, j)) F_{0}(y, j)
$$

for any $t \geq 0$ and $(x, i) \in X_{4}$. Note that $F_{t} \in X_{5}$ for any $t \geq 0$ following from Theorem 9.3.1 of Liggett (1985). Since $G_{\lambda}((x, i),(y, j)) \geq 0$ when $(x, i) \neq(y, j)$, it is easy to check that

$$
e^{t G_{\lambda}}((x, i),(y, j)) \geq 0
$$


for any $(x, i),(y, j) \in X_{4}$. Then, according to Equations (4.10), (4.11) and the fact that $F_{0}(x, i)=1$ for any $(x, i) \in X_{4}$,

$$
\begin{aligned}
E_{\lambda}\left(\zeta_{t}(O) \zeta_{t}(x)\right) & =F_{t}(x, 1)=\sum_{(y, j) \in X_{4}} e^{t G_{\lambda}}((x, 1),(y, j)) \\
& \leq \sum_{(y, j) \in X_{4}} e^{t G_{\lambda}}((x, 1),(y, j)) \frac{K_{\lambda}(y, j)}{\inf _{(u, i) \in X_{4}} K_{\lambda}(u, i)} \\
& =\frac{K_{\lambda}(x, 1)}{\inf _{(u, i) \in X_{4}} K_{\lambda}(u, i)}<+\infty
\end{aligned}
$$

for any $t \geq 0$. Let $x=O$, then Lemma 4.4 follows from Equation (4.12) directly.

By Lemma 4.4, we want to find $\lambda$ which ensures the existence of the positive eigenvector $K_{\lambda}$ of $G_{\lambda}$ with respect to the eigenvalue 0 . For this purpose, we need two random walks. We denote by $\left\{S_{n}\right\}_{n \geq 0}$ the simple random walk on $\mathbb{Z}^{d}$ such that

$$
P\left(S_{n+1}=y \mid S_{n}=x\right)=\frac{1}{2 d}
$$

for any $n \geq 0$ and $x, y \in \mathbb{Z}^{d}, x \sim y$. Let $\left\{\theta_{n}\right\}_{n \geq 0}$ be a random walk on

$$
X_{4} \backslash\{(O, 3)\}=\left\{(x, i): x \in \mathbb{Z}^{d}, i \in\{1,2,3\} \text { and }(x, i) \neq(O, 3)\right\}
$$

such that for each $n \geq 0$,

$$
\begin{aligned}
& P\left(\theta_{n+1}=(y, j) \mid \theta_{n}=(x, i)\right)= \\
& \begin{cases}1 & \text { if } x \neq O, i=1 \text { and }(y, j)=(x, 2), \\
\frac{1}{2+\delta+\gamma} & \text { if } x \neq O, i=2 \text { and }(y, j)=(x, 3), \\
\frac{1}{2 d} \frac{1+\delta+\gamma}{2+\delta+\gamma} & \text { if } x \neq O, i=2, y \sim x \text { and } j=1, \\
\frac{1}{2 d} & \text { if } x \neq O, i=3, y \sim x \text { and } j=2, \\
1 & \text { if }(x, i)=(y, j)=(O, 1), \\
1 & \text { if }(x, i)=(O, 2) \text { and }(y, j)=\left(e_{1}, 1\right), \\
0 & \text { else, }\end{cases}
\end{aligned}
$$

then we define

$$
\Gamma(x, i)=P\left(\theta_{n}=(O, 1) \text { for some } n \geq 0 \mid \theta_{0}=(x, i)\right)
$$

for $(x, i) \in X_{4}$ such that $(x, i) \neq(O, 3)$, i.e., $\Gamma(x, i)$ is the probability that $\left\{\theta_{n}\right\}_{n \geq 0}$ visits $(O, 1)$ at least once conditioned on $\theta_{0}=(x, i)$. By the definition of $\left\{\theta_{t}\right\}_{t \geq 0}$ and the strong Markov property, $\Gamma(x, i)$ satisfies

$$
\begin{aligned}
\Gamma(x, 1) & =\Gamma(x, 2) \text { if } x \neq O, \\
\Gamma(x, 2) & =\frac{1}{2+\delta+\gamma+\lambda} \Gamma(x, 3)+\frac{1}{2 d} \frac{1+\delta+\gamma+\lambda}{2+\delta+\gamma+\lambda} \sum_{y: y \sim x} \Gamma(y, 1) \text { if } x \neq O, \\
\Gamma(x, 3) & =\frac{1}{2 d} \sum_{y: y \sim x} \Gamma(y, 2) \text { if } x \neq O, \\
\Gamma(O, 2) & =\Gamma\left(e_{1}, 1\right) \text { and } \Gamma(O, 1)=1 .
\end{aligned}
$$


For any $x \in \mathbb{Z}^{d}$, we define

$$
\widetilde{\Gamma}(x)=P\left(S_{n}=O \text { for some } n \geq 0 \mid S_{0}=x\right)
$$

as the probability that $\left\{S_{n}\right\}_{n \geq 0}$ visits $O$ at least once conditioned on $S_{0}=x$. We claim that

$$
\Gamma(x, 1) \leq \widetilde{\Gamma}(x)
$$

for $x \neq O$. Equation (4.14) follows from the following analysis. For each $n \geq 0$, we write $\theta_{n}$ as $\left(\theta_{n}(1), \theta_{n}(2)\right)$ such that $\theta_{n}(1) \in \mathbb{Z}^{d}$ and $\theta_{n}(2) \in\{0,1,2\}$. Conditioned on $\theta_{0}=(x, 1)$ with $x \neq O,\left\{\theta_{n}(1)\right\}_{n \geq 0}$ is a lazy version of $\left\{S_{n}\right\}_{n \geq 1}$ with $S_{0}=x$ until the first moment $n_{0}$ that $\theta_{n_{0}}(1)=O$ according to the definition of $\left\{\theta_{n}\right\}_{n \geq 0}$. In other words, before hitting $O, \theta(1)$ chooses each neighbor to jump with the same probability $\frac{1}{2 d}$ when $\theta(1)$ jumps at some steps while $\theta(1)$ stays still at other steps. Therefore,

$$
\begin{aligned}
\Gamma(x, 1) & =P\left(\theta_{n}=(O, 1) \text { for some } n \geq 0 \mid \theta_{0}=(x, 1)\right) \\
& \leq P\left(\theta_{n}(1)=O \text { for some } n \geq 0 \mid \theta_{0}=(x, 1)\right) \\
& =P\left(S_{n}=O \text { for some } n \geq 0 \mid S_{0}=x\right)=\widetilde{\Gamma}(x)
\end{aligned}
$$

and hence Equation (4.14) holds. According to the result given in Kesten (1964) authored by Kesten,

$$
\widetilde{\Gamma}\left(e_{1}\right)=\frac{1}{2 d}+\frac{1}{2 d^{2}}+O\left(\frac{1}{d^{3}}\right)
$$

as the dimension $d$ of the lattice grows to infinity. An intuitive explanation of the above equation is that the random walk either visits $O$ in the first step, or it never goes there. By Equation (4.15),

$$
\gamma-(2 \gamma+2) \widetilde{\Gamma}\left(e_{1}\right)>0
$$

when the dimension $d$ of the lattice is sufficiently large. Now we can give the proof of Equation (2.6).

Proof of Equation (2.6): We assume that the dimension $d$ of the lattice is sufficiently large such that Equation (4.16) holds, then we define

$$
\widetilde{\lambda}=\frac{1+\delta+\gamma}{2 d\left[\gamma-(2 \gamma+2) \widetilde{\Gamma}\left(e_{1}\right)\right]},
$$

which is positive. Furthermore, we define

$$
h_{\lambda}=\frac{\gamma[1-2 \Gamma(O, 2)]-2 \Gamma\left(e_{1}, 2\right)-\frac{1+\delta+\gamma}{2 d \lambda}}{\gamma+2+\frac{1+\delta+\gamma}{2 d \lambda}} .
$$

According to Equation (4.14) and the fact that $\Gamma(O, 2)=\Gamma\left(e_{1}, 1\right)$ while

$$
\Gamma(x, 1)=\Gamma(x, 2)
$$

for $x \neq O$, it is easy to check that $h_{\lambda}>0$ when $\lambda>\widetilde{\lambda}$. For any $\lambda>\widetilde{\lambda}$, we define $K_{\lambda}: X_{4} \rightarrow[0,+\infty)$ as

$$
K_{\lambda}(x, i)= \begin{cases}\Gamma(x, i)+h_{\lambda} & \text { if }(x, i) \neq(O, 3), \\ \gamma\left[1-2 \Gamma\left(e_{1}, 1\right)-h_{\lambda}\right] & \text { if }(x, i)=(O, 3) .\end{cases}
$$


Since $h_{\lambda} \leq 1-2 \Gamma(O, 2)=1-2 \Gamma\left(e_{1}, 1\right)$,

$$
\inf _{(x, i) \in X_{4}} K_{\lambda}(x, i) \geq \inf \left\{h_{\lambda}, \gamma\left[1-2 \Gamma\left(e_{1}, 1\right)-h_{\lambda}\right]\right\}>0
$$

for $\lambda>\widetilde{\lambda}$. By the definition of $G_{\lambda}$ and Equation (4.13), it is easy to check that

$$
G_{\lambda} K_{\lambda}=0 \text { (zero function) }
$$

by direct calculation. Then, by Lemma 4.4 and Equation (4.17),

$$
\sup _{t \geq 0} E_{\lambda}\left(\zeta_{t}^{2}(O)\right)<+\infty
$$

when $\lambda>\tilde{\lambda}$. Therefore, by Lemma 4.2 ,

$$
\lambda_{c} \leq \lambda
$$

for any $\lambda>\widetilde{\lambda}$ and hence

$$
\lambda_{c} \leq \widetilde{\lambda}=\frac{1+\delta+\gamma}{2 d\left[\gamma-(2 \gamma+2) \widetilde{\Gamma}\left(e_{1}\right)\right]} .
$$

By utilizing the fact that $\frac{1}{1-x}=\sum_{n=0}^{+\infty} x^{n}$ for $x \in(0,1)$, Equation (2.6) follows directly from Equations (4.15) and (4.18).

\section{Upper bounds on $1-\pi(A, B)$}

In this section we will prove the following lemma, which gives upper bounds on $1-\pi(A, B)$.

Lemma 5.1. For any $\lambda>\frac{1+\gamma+\delta}{\gamma}, d \geq 1, m, n \geq 0$ and $A, B \subseteq \mathbb{Z}^{d}$ such that $|A|=m,|B|=n$ while $A \cap B=\emptyset$,

$$
1-\pi\left(A, B, \frac{\lambda}{2 d}, d\right) \leq 1-\left(1-\frac{\lambda \gamma-(1+\delta+\gamma)}{\lambda(\gamma+1)}\right)^{m}\left(\frac{1+\delta+\gamma}{\lambda \gamma}\right)^{n} .
$$

After reading the next section, readers can find that the bound in Lemma 5.1 is sharp in the limit $d \rightarrow+\infty$.

To prove Lemma 5.1, we need two auxiliary processes. The first is the 'on-off' process introduced in Krone (1999). The second is a two-type branching process. The 'on-off' process $\left\{\xi_{t}\right\}_{t \geq 0}$ is a continuous-time Markov process with state space $\{0,1,2\}^{\mathbb{Z}^{d}}$ and transition rates function given as follows. For each $x \in \mathbb{Z}^{d}$ and $t \geq 0$,

$$
\begin{aligned}
& \xi_{t}(x) \text { flips from } i \text { to } j \text { at rate } \\
& \begin{cases}1 & \text { if } i \in\{1,2\} \text { and } j=0, \\
\delta & \text { if } i=2 \text { and } j=1, \\
\gamma & \text { if } i=1 \text { and } j=2, \\
\lambda \sum_{y \sim x} 1_{\left\{\xi_{t}(y)=2\right\}} & \text { if } i=0 \text { and } j=1, \\
0 & \text { otherwise, }\end{cases}
\end{aligned}
$$

where $\lambda, \delta, \gamma$ are constants defined as in Equation (1.1).

For any $t \geq 0$, we define $\widehat{C}_{t}=\left\{x: \xi_{t}(x)=2\right\}$ and $\widehat{D}_{t}=\left\{x: \xi_{t}(x)=1\right\}$. We write $\xi_{t}, \widehat{C}_{t}, \widehat{D}_{t}$ as $\xi_{t}^{(C, D)}, \widehat{C}_{t}^{(C, D)}, \widehat{D}_{t}^{(C, D)}$ when $\widehat{C}_{0}=C$ and $\widehat{D}_{0}=D$. 
By utilizing the graphical representation of the two-stage contact process introduced in Krone (1999), which we reviewed in Section 3, Krone gives the following duality relationship between the two-stage contact process $\left\{\eta_{t}\right\}_{t \geq 0}$ and the 'on-off' process $\left\{\xi_{t}\right\}_{t \geq 0}$ with identical parameters $\lambda, \delta, \gamma$.

Proposition 5.2. (Krone, 1999) For any $A, B, C, D \subseteq \mathbb{Z}^{d}$ such that $A \cap B=\emptyset$ and $C \cap D=\emptyset$,

$$
\begin{aligned}
& P_{\lambda}\left(\eta_{t}^{(C, D)}(x)=2 \text { for some } x \in A \text { or } \eta_{t}^{(C, D)}(y) \neq 0 \text { for some } y \in B\right) \\
& =P_{\lambda}\left(\xi_{t}^{(B, A)}(x)=2 \text { for some } x \in D \text { or } \xi_{t}^{(B, A)}(y) \neq 0 \text { for some } y \in C\right) .
\end{aligned}
$$

If we let $C=\mathbb{Z}^{d}$ and $D=\emptyset$ while we let $t$ grow to infinity, then we have the following direct corollary.

Corollary 5.3. (Krone, 1999) For any $A, B \subseteq \mathbb{Z}^{d}$ such that $A \cap B=\emptyset$,

$$
1-\pi(A, B, \lambda, d)=P_{\lambda}\left(\widehat{C}_{t}^{(B, A)} \cup \widehat{D}_{t}^{(B, A)} \neq \emptyset \text { for all } t \geq 0\right)
$$

To bound $P_{\lambda}\left(\widehat{C}_{t}^{(B, A)} \cup \widehat{D}_{t}^{(B, A)} \neq \emptyset\right.$ for all $\left.t \geq 0\right)$ from above, we introduce a two-type branching process where there are some type 1 individuals and some type 2 individuals at $t=0$. Each individual is independently removed from the system at rate 1 . Each type 1 individual independently becomes a type 2 individual at rate $\gamma$. Each type 2 individual independently becomes a type 1 individual at rate $\delta$ while it gives birth to a type 1 individual at rate $\lambda$.

That is to say, if we use $\widehat{\zeta}_{t}$ to denote the number of type 2 individuals at $t$ while use $\widehat{g}_{t}$ to denote the number of type 1 individuals at $t$, then $\left\{\left(\widehat{\zeta}_{t}, \widehat{g}_{t}\right)\right\}_{t \geq 0}$ evolves as follows.

$$
\left(\widehat{\zeta}_{t}, \widehat{g}_{t}\right) \text { flips to } \begin{cases}\left(\widehat{\zeta}_{t}-1, \widehat{g}_{t}\right) & \text { at rate } \widehat{\zeta}_{t}, \\ \left(\widehat{\zeta}_{t}, \widehat{g}_{t}-1\right) & \text { at rate } \widehat{g}_{t}, \\ \left(\widehat{\zeta}_{t}+1, \widehat{g}_{t}-1\right) & \text { at rate } \gamma \widehat{g}_{t}, \\ \left(\widehat{\zeta}_{t}-1, \widehat{g}_{t}+1\right) & \text { at rate } \delta \widehat{\zeta}_{t}, \\ \left(\widehat{\zeta}_{t}, \widehat{g}_{t}+1\right) & \text { at rate } \lambda \widehat{\zeta}_{t}, \\ 0 & \text { otherwise. }\end{cases}
$$

For $m, n \geq 0$, we use $\widehat{\pi}(n, m)$ to denote the probability of the event that $\widehat{\zeta}_{t}+\widehat{g}_{t}>0$ for all $t \geq 0$ conditioned on there being $n$ type 2 individuals and $m$ type 1 individuals at $t=0$. We write $\widehat{\pi}(n, m)$ as $\widehat{\pi}(n, m, \lambda)$ when we need to point out the rate $\lambda$ at which a type 2 individual gives birth to a type 1 individual. Then, we have the following lemma.

Lemma 5.4. For any $m, n \geq 0$ and $\lambda>\frac{1+\delta+\gamma}{\gamma}$,

$$
\widehat{\pi}(n, m, \lambda)=1-\left(\frac{1+\delta+\gamma}{\lambda \gamma}\right)^{n}\left(1-\frac{\lambda \gamma-(1+\delta+\gamma)}{\lambda(\gamma+1)}\right)^{m}
$$

Proof: According to the property of independent exponential times and the strong Markov property,

$$
\begin{cases}\widehat{\pi}(1,0) & =\frac{\lambda}{1+\delta+\lambda} \widehat{\pi}(1,1)+\frac{\delta}{1+\delta+\lambda} \widehat{\pi}(0,1) \\ \widehat{\pi}(0,1) & =\frac{\gamma}{1+\gamma} \widehat{\pi}(1,0)\end{cases}
$$


Since the activities of different individuals are independent, for any $m, n \geq 0$,

$$
\widehat{\pi}(n, m)=1-(1-\widehat{\pi}(1,0))^{n}(1-\widehat{\pi}(0,1))^{m} .
$$

Applying Equations (5.3) and (5.4) with $m=n=1$, we have

$$
\widehat{\pi}(1,0)[\lambda \gamma(1-\widehat{\pi}(1,0))-(1+\delta+\gamma)]=0
$$

By direct calculation, when $\lambda>\frac{1+\delta+\gamma}{\gamma}$, the mean of the number of type 2 children of a type 2 father is

$$
\frac{\lambda \gamma}{1+\delta+\gamma}>1
$$

Therefore, $\widehat{\pi}(1,0, \lambda)>0$ when $\lambda>\frac{1+\delta+\gamma}{\gamma}$ according to the classic theory of branching processes (See Theorem 3.1 of the book van der Hofstad (2017) authored by van der Hofstad). Then, by Equation (5.5),

$$
\widehat{\pi}(1,0, \lambda)=1-\frac{1+\delta+\gamma}{\lambda \gamma}
$$

when $\lambda>\frac{1+\delta+\gamma}{\gamma}$. As a result, by Equations (5.3) and (5.4),

$$
\widehat{\pi}(0,1, \lambda)=\frac{\lambda \gamma-(1+\delta+\gamma)}{\lambda(\gamma+1)}
$$

and

$$
\widehat{\pi}(n, m, \lambda)=1-\left(\frac{1+\delta+\gamma}{\lambda \gamma}\right)^{n}\left(1-\frac{\lambda \gamma-(1+\delta+\gamma)}{\lambda(\gamma+1)}\right)^{m}
$$

for any $m, n \geq 0$ and $\lambda>\frac{1+\delta+\gamma}{\gamma}$.

Now we can give the proof of Lemma 5.1.

Proof of Lemma 5.1: For the 'on-off' process $\left\{\xi_{t}\right\}_{t \geq 0}$ on $\mathbb{Z}^{d}$ with parameter $\frac{\lambda}{2 d}, \delta, \gamma$, a type 2 vertex gives birth to a type 1 vertex at rate

$$
\frac{\lambda}{2 d} \sum_{y \sim x} 1_{\left\{\xi_{t}(y)=0\right\}} \leq \frac{\lambda}{2 d} \times 2 d=\lambda .
$$

As a result, for $A, B \subseteq \mathbb{Z}^{d}$ such that $|A|=m$ and $|B|=n$ while $A \cap B=\emptyset,\left|\widehat{C}_{t}^{(B, A)}\right|$ and $\left|\widehat{D}_{t}^{(B, A)}\right|$ are stochastically dominated from above by the numbers of type 2 individuals and type 1 individuals at moment $t$ respectively of the two-type branching process with $n$ initial type 2 individuals and $m$ initial type 1 individuals. In detail, conditioned on $\widehat{\zeta}_{0}=n$ and $\widehat{g}_{0}=m,\left\{\left(\left|\widehat{C}_{t}^{(B, A)}\right|,\left|\widehat{D}_{t}^{(B, A)}\right|\right)\right\}_{t \geq 0}$ and $\left\{\left(\widehat{\zeta}_{t}, \widehat{g}_{t}\right)\right\}_{t \geq 0}$ can be coupled in the following way, which is a so called 'Vasershtein coupling' (see 
page 124 of Liggett, 1985). For any $t \geq 0,\left(\left|\widehat{C}_{t}^{(B, A)}\right|,\left|\widehat{D}_{t}^{(B, A)}\right|, \widehat{\zeta}_{t}, \widehat{g}_{t}\right)$ flips to

$$
\left\{\begin{array}{l}
\left(\left|\widehat{C}_{t}^{(B, A)}\right|-1,\left|\widehat{D}_{t}^{(B, A)}\right|, \widehat{\zeta}_{t}-1, \widehat{g}_{t}\right) \text { at rate }\left|\widehat{C}_{t}^{(B, A)}\right|, \\
\left(\left|\widehat{C}_{t}^{(B, A)}\right|,\left|\widehat{D}_{t}^{(B, A)}\right|, \widehat{\zeta}_{t}-1, \widehat{g}_{t}\right) \text { at rate } \widehat{\zeta}_{t}-\left|\widehat{C}_{t}^{(B, A)}\right|, \\
\left(\left|\widehat{C}_{t}^{(B, A)}\right|,\left|\widehat{D}_{t}^{(B, A)}\right|-1, \widehat{\zeta}_{t}, \widehat{g}_{t}-1\right) \text { at rate }\left|\widehat{D}_{t}^{(B, A)}\right|, \\
\left(\left|\widehat{C}_{t}^{(B, A)}\right|,\left|\widehat{D}_{t}^{(B, A)}\right|, \widehat{\zeta}_{t}, \widehat{g}_{t}-1\right) \text { at rate } \widehat{g}_{t}-\left|\widehat{D}_{t}^{(B, A)}\right|, \\
\left(\left|\widehat{C}_{t}^{(B, A)}\right|+1,\left|\widehat{D}_{t}^{(B, A)}\right|-1, \widehat{\zeta}_{t}+1, \widehat{g}_{t}-1\right) \text { at rate } \gamma\left|\widehat{D}_{t}^{(B, A)}\right|, \\
\left.\left|\widehat{C}_{t}^{(B, A)}\right|,\left|\widehat{D}_{t}^{(B, A)}\right|, \widehat{\zeta}_{t}+1, \widehat{g}_{t}-1\right) \text { at rate } \gamma\left(\widehat{g}_{t}-\left|\widehat{D}_{t}^{(B, A)}\right|\right), \\
\left(\left|\widehat{C}_{t}^{(B, A)}\right|-1,\left|\widehat{D}_{t}^{(B, A)}\right|+1, \widehat{\zeta}_{t}-1, \widehat{g}_{t}+1\right) \text { at rate } \delta\left|\widehat{C}_{t}^{(B, A)}\right|, \\
\left(\left|\widehat{C}_{t}^{(B, A)}\right|,\left|\widehat{D}_{t}^{(B, A)}\right|, \widehat{\zeta}_{t}-1, \widehat{g}_{t}+1\right) \text { at rate } \delta\left(\widehat{\zeta}_{t}-\left|\widehat{C}_{t}^{(B, A)}\right|\right), \\
\left(\left|\widehat{C}_{t}^{(B, A)}\right|,\left|\widehat{D}_{t}^{(B, A)}\right|+1, \widehat{\zeta}_{t}, \widehat{g}_{t}+1\right) \text { at rate } \frac{\lambda \sum_{x \in \widehat{C}_{t}^{(B, A)} \sum_{y \sim x} 1_{\left\{\xi_{t}(y)=0\right\}}}^{2 d},}{\left(\left|\widehat{C}_{t}^{(B, A)}\right|,\left|\widehat{D}_{t}^{(B, A)}\right|, \widehat{\zeta}_{t}, \widehat{g}_{t}+1\right) \text { at rate } \lambda \widehat{\zeta}_{t}-\frac{\lambda \sum_{x \in \widehat{C}_{t}^{(B, A)} \sum_{y \sim x} 1_{\left\{\xi_{t}(y)=0\right\}}}}{2 d}} .
\end{array}\right.
$$

Note that the above coupling maintains the property that $\left|\widehat{C}_{t}^{(B, A)}\right| \leq \widehat{\zeta}_{t}$ and $\left|\widehat{D}_{t}^{(B, A)}\right| \leq \widehat{g}_{t}$ for all $t \geq 0$. Therefore,

$$
P_{\frac{\lambda}{2 d}, d}\left(\widehat{C}_{t}^{(B, A)} \cup \widehat{D}_{t}^{(B, A)} \neq \emptyset \text { for all } t \geq 0\right) \leq \widehat{\pi}(n, m, \lambda) .
$$

Lemma 5.1 follows from Corollary 5.3, Lemma 5.4 and Equation (5.7) directly.

\section{Lower bounds on $1-\pi(A, B)$}

In this section we will give lower bounds on $1-\pi(A, B)$ to complete the proof of Theorem 2.2. First we introduce some notation and definitions for later use. Let $\widetilde{X}_{1}, \ldots, \widetilde{X}_{n}, \ldots$ be independent and identically distributed Bernoulli random variables such that

$$
P\left(\widetilde{X}_{1}=1\right)=e^{-(1+\delta)}\left(1-e^{-\gamma}\right)=1-P\left(\widetilde{X}_{1}=0\right),
$$

then, for each integer $M \geq 1$, we define

$$
\widetilde{\alpha}(M)=P\left(\frac{\sum_{i=1}^{M} \widetilde{X}_{i}}{M} \geq \frac{e^{-(1+\delta)}\left(1-e^{-\gamma}\right)}{2}\right) .
$$

For any $d \geq 1, \lambda>0$ and $n \geq 1$, we define

$$
\begin{gathered}
\tilde{b}(d, n, \lambda)=\inf \left\{\nu_{\frac{\lambda}{2 d}}, d(\eta(x) \neq 0 \text { for some } x \in A):\right. \\
\left.A \subseteq \mathbb{Z}^{d} \text { and }|A|=n\right\} .
\end{gathered}
$$

The aim of this section is to prove the following two lemmas.

Lemma 6.1. For $\lambda>\frac{1+\gamma+\delta}{\gamma}$ and $n \geq 1$,

$$
\liminf _{d \rightarrow+\infty} \tilde{b}(d, n, \lambda) \geq \frac{1}{\frac{1}{n} \frac{2(\gamma+1)}{\gamma-\frac{1+\delta+\gamma}{\lambda}}+\frac{n-1}{n}} .
$$


Lemma 6.2. For $m, n \geq 0, \lambda>\frac{1+\delta+\gamma}{\gamma}, M>n+m$ and sufficiently large $d$,

$$
\begin{aligned}
1-\pi\left(A, B, \frac{\lambda}{2 d}, d\right) \geq & \left\{1-\left(\frac{1+\delta+\gamma}{\frac{(2 d-M) \lambda}{2 d} \gamma}\right)^{n}\left(1-\frac{\frac{(2 d-M) \lambda}{2 d} \gamma-(1+\delta+\gamma)}{\frac{(2 d-M) \lambda}{2 d}(\gamma+1)}\right)^{m}\right\} \\
& \times \widetilde{\alpha}(M) \widetilde{b}\left(d,\left\lceil\frac{M e^{-(1+\delta)}\left(1-e^{-\gamma}\right)}{2}\right\rceil, \lambda\right)
\end{aligned}
$$

for any $A, B \subseteq \mathbb{Z}^{d}$ such that $|A|=m,|B|=n$ and $A \cap B=\emptyset$, where

$$
\lceil x\rceil=\inf \{m: m \geq x \text { and } m \text { is an integer }\} .
$$

Note that the right-hand side of the inequality in Lemma 6.1 approaches 1 as $n \rightarrow+\infty$.

Before proving Lemmas 6.1 and 6.2, we first show how to utilize these two lemmas to prove Theorem 2.2.

Proof of Theorem 2.2: For simplicity, we use $\widetilde{c}(M, d, \lambda)$ to denote

$$
\begin{aligned}
& \left\{1-\left(\frac{1+\delta+\gamma}{\frac{(2 d-M) \lambda}{2 d} \gamma}\right)^{n}\left(1-\frac{\frac{(2 d-M) \lambda}{2 d} \gamma-(1+\delta+\gamma)}{\frac{(2 d-M) \lambda}{2 d}(\gamma+1)}\right)^{m}\right\} \\
& \times \widetilde{\alpha}(M) \widetilde{b}\left(d,\left\lceil\frac{M e^{-(1+\delta)}\left(1-e^{-\gamma}\right)}{2}\right\rceil, \lambda\right)
\end{aligned}
$$

while use $\mu(M)$ to denote $\left\lceil\frac{M e^{-(1+\delta)}\left(1-e^{-\gamma}\right)}{2}\right\rceil$. As we have defined in Section 2,

$$
\begin{aligned}
& \Pi(m, n, \lambda, d)= \\
& \sup \left\{\left|\pi\left(A, B, \frac{\lambda}{2 d}, d\right)-\left(1-\frac{\lambda \gamma-(1+\delta+\gamma)}{\lambda(\gamma+1)}\right)^{m}\left(\frac{1+\delta+\gamma}{\lambda \gamma}\right)^{n}\right|:\right. \\
& \left.A, B \subseteq \mathbb{Z}^{d},|A|=m,|B|=n, A \cap B=\emptyset\right\} .
\end{aligned}
$$

Then, according to Lemmas 5.1 and 6.2,

$$
\Pi(m, n, \lambda, d) \leq 1-\left(1-\frac{\lambda \gamma-(1+\delta+\gamma)}{\lambda(\gamma+1)}\right)^{m}\left(\frac{1+\delta+\gamma}{\lambda \gamma}\right)^{n}-\widetilde{c}(M, d, \lambda)
$$

for $m, n \geq 0$ and $\lambda>\frac{1+\delta+\gamma}{\gamma}$. By Lemma 6.1,

$$
\begin{aligned}
\liminf _{d \rightarrow+\infty} \widetilde{c}(M, d, \lambda) \geq & \left\{1-\left(1-\frac{\lambda \gamma-(1+\delta+\gamma)}{\lambda(\gamma+1)}\right)^{m}\left(\frac{1+\delta+\gamma}{\lambda \gamma}\right)^{n}\right\} \\
& \times \widetilde{\alpha}(M) \frac{1}{\frac{2(\gamma+1)}{\mu(M)\left(\gamma-\frac{1+\delta+\gamma}{\lambda}\right)}+\frac{\mu(M)-1}{\mu(M)}}
\end{aligned}
$$


for sufficiently large $M$ such that $\mu(M)>1$ and $\lambda>\frac{1+\delta+\gamma}{\gamma}$. By Equations (6.1) and (6.2),

$$
\begin{aligned}
\limsup _{d \rightarrow+\infty} \Pi(m, n, \lambda, d) \leq & \left\{1-\left(1-\frac{\lambda \gamma-(1+\delta+\gamma)}{\lambda(\gamma+1)}\right)^{m}\left(\frac{1+\delta+\gamma}{\lambda \gamma}\right)^{n}\right\} \\
& \times\left(1-\widetilde{\alpha}(M) \frac{1}{\frac{2(\gamma+1)}{\mu(M)\left(\gamma-\frac{1+\delta+\gamma}{\lambda}\right)}+\frac{\mu(M)-1}{\mu(M)}}\right)
\end{aligned}
$$

for any sufficiently large $M$ and $\lambda>\frac{1+\delta+\gamma}{\gamma}$. According to the weak law of large numbers (See Theorem 2.2.9 of Durrett, 2010),

$$
\lim _{M \rightarrow+\infty} \widetilde{\alpha}(M)=1 \text {. }
$$

As a result, let $M \rightarrow+\infty$,

$$
\limsup _{d \rightarrow+\infty} \Pi(m, n, \lambda, d) \leq 0
$$

for any $\lambda>\frac{1+\delta+\gamma}{\gamma}$ according to Equation (6.3) and the fact that

$$
\lim _{M \rightarrow+\infty} \mu(M)=+\infty \text {. }
$$

Since $\Pi(m, n, \lambda, d)$ is nonnegative, Theorem 2.2 follows from Equation (6.4) directly.

Now we give the proof of Lemma 6.1.

Proof of Lemma 6.1: Let $A \subseteq \mathbb{Z}^{d}$ such that $|A|=n$, then according to the definition of $\nu$ and Cauchy-Schwartz's inequality,

$$
\begin{aligned}
& \nu_{\frac{\lambda}{2 d}, d}(\eta(x) \neq 0 \text { for some } x \in A) \\
& =\lim _{t \rightarrow+\infty} P_{\frac{\lambda}{2 d}, d}\left(\eta_{t}^{\left(\mathbb{Z}^{d}, \emptyset\right)}(x) \neq 0 \text { for some } x \in A\right) \\
& =\lim _{t \rightarrow+\infty} P_{\frac{\lambda}{2 d}, d}\left(\zeta_{t}(x)+g_{t}(x)>0 \text { for some } x \in A\right) \\
& \geq \limsup _{t \rightarrow+\infty} P_{\frac{\lambda}{2 d}, d}\left(\zeta_{t}(x)>0 \text { for some } x \in A\right) \\
& =\limsup _{t \rightarrow+\infty} P_{\frac{\lambda}{2 d}, d}\left(\sum_{x \in A} \zeta_{t}(x)>0\right) \geq \limsup _{t \rightarrow+\infty} \frac{\left(E_{\frac{\lambda}{2 d}, d} \sum_{x \in A} \zeta_{t}(x)\right)^{2}}{E_{\frac{\lambda}{2 d}}\left(\left(\sum_{x \in A} \zeta_{t}(x)\right)^{2}\right)},
\end{aligned}
$$

where $\left\{\left(\zeta_{t}(x), g_{t}(x)\right): t \geq 0, x \in \mathbb{Z}^{d}\right\}$ is our auxiliary model defined as in Section 4 . We have shown in Section 4 that $E \zeta_{t}(x)=E \zeta_{t}(O) \equiv 1$, then by Equation (6.5),

$$
\begin{aligned}
& \nu_{\frac{\lambda}{2 d}, d}(\eta(x) \neq 0 \text { for some } x \in A) \geq \limsup _{t \rightarrow+\infty} \frac{1}{\frac{1}{n^{2}} \sum_{x, y \in A} F_{t}(y-x, 1)} \\
& =\limsup _{t \rightarrow+\infty} \frac{1}{\frac{1}{n} F_{t}(O, 1)+\frac{1}{n^{2}} \sum_{x, y \in A, x \neq y} F_{t}(y-x, 1)},
\end{aligned}
$$

where $F_{t}(x, 1)=E_{\frac{\lambda}{2 d}, d}\left(\zeta_{t}(O) \zeta_{t}(x)\right)=E_{\frac{\lambda}{2 d}, d}\left(\zeta_{t}(y) \zeta_{t}(x+y)\right)$ defined as in Section 4 . For $\lambda>\frac{1+\delta+\gamma}{\gamma}$ and sufficiently large $d$, let $K_{\frac{\lambda}{2 d}}$ be the function from $X_{4}$ to $\mathbb{R}$ 
defined as before equation (4.17). Then, $K_{\frac{\lambda}{2 d}}$ is the eigenvector with respect to the eigenvalue 0 of the $X_{4} \times X_{4}$ matrix $G_{\frac{\lambda}{2 d}}$ and

$$
F_{t}(x, 1) \leq \frac{K_{\frac{\lambda}{2 d}}(x, 1)}{\inf _{(u, i) \in X_{4}} K_{\frac{\lambda}{2 d}}(u, i)}
$$

as we have shown in Equation (4.12) and the proof of Equation (2.6). Note that $\inf _{(x, i) \in X_{4}} K_{\frac{\lambda}{2 d}}(x, i)>0$ when $\lambda>\frac{1+\gamma+\delta}{\gamma}$ and $d$ is sufficiently large according to the definition of $K_{\frac{\lambda}{2 d}}$ and the fact that

$$
\Gamma\left(e_{1}, 1\right) \leq \widetilde{\Gamma}\left(e_{1}\right)=\frac{1}{2 d}+O\left(\frac{1}{d^{2}}\right) .
$$

As we have defined in Section 4,

$$
K_{\frac{\lambda}{2 d}}(x, 1)=\Gamma(x, 1)+h_{\frac{\lambda}{2 d}},
$$

where

$$
h_{\frac{\lambda}{2 d}}=\frac{\gamma[1-2 \Gamma(O, 2)]-2 \Gamma\left(e_{1}, 2\right)-\frac{1+\delta+\gamma}{\lambda}}{\gamma+2+\frac{1+\delta+\gamma}{\lambda}} .
$$

By Equation (4.17),

$$
\inf _{(x, i) \in X_{4}} K_{\frac{\lambda}{2 d}}(x, i) \geq \inf \left\{h_{\frac{\lambda}{2 d}}, \gamma\left[1-2 \Gamma\left(e_{1}, 1\right)-h_{\frac{\lambda}{2 d}}\right]\right\} .
$$

Then, according to the definition of $h_{\lambda}$ and the fact that $\Gamma\left(e_{1}, 1\right) \leq \widetilde{\Gamma}\left(e_{1}\right)=\frac{1}{2 d}+$ $O\left(\frac{1}{d^{2}}\right)$,

and hence

$$
\gamma\left[1-2 \Gamma\left(e_{1}, 1\right)-h_{\frac{\lambda}{2 d}}\right]>h_{\frac{\lambda}{2 d}}
$$

$$
\inf _{(x, i) \in X_{4}} K_{\frac{\lambda}{2 d}}(x, i) \geq h_{\frac{\lambda}{2 d}}
$$

for sufficiently large $d$. By Equations (6.7) and (6.8), for sufficiently large $d$,

$$
F_{t}(x, 1) \leq \frac{\Gamma(x, 1)+h_{\frac{\lambda}{2 d}}}{h_{\frac{\lambda}{2 d}}} \leq \frac{\widetilde{\Gamma}(x)+h_{\frac{\lambda}{2 d}}}{h_{\frac{\lambda}{2 d}}} \leq \frac{\widetilde{\Gamma}\left(e_{1}\right)+h_{\frac{\lambda}{2 d}}}{h_{\frac{\lambda}{2 d}}}
$$

for any $x \neq O$ while

$$
F_{t}(O, 1) \leq \frac{1+h_{\frac{\lambda}{2 d}}}{h_{\frac{\lambda}{2 d}}}
$$

By Equations (6.6), (6.9) and (6.10),

$$
\nu_{\frac{\lambda}{2 d}, d}(\eta(x) \neq 0 \text { for some } x \in A) \geq \frac{1}{\frac{1}{n} \frac{1+h_{\frac{\lambda}{2 d}}}{h_{\frac{\lambda}{2 d}}}+\frac{n(n-1)}{n^{2}} \frac{\widetilde{\Gamma}\left(e_{1}\right)+h_{\frac{\lambda}{2 d}}}{h_{\frac{\lambda}{2 d}}}} .
$$

Since $\widetilde{\Gamma}\left(e_{1}\right)=\frac{1}{2 d}+O\left(\frac{1}{d^{2}}\right)$ and

$$
\lim _{d \rightarrow+\infty} h_{\frac{\lambda}{2 d}}=\frac{\gamma-\frac{1+\delta+\gamma}{\lambda}}{\gamma+2+\frac{1+\delta+\gamma}{\lambda}}
$$

Lemma 6.1 follows directly from Equation (6.11).

At last, we only need to give the proof of Lemma 6.2. Before jumping into the proof, we explain the meaning of each term in Lemma 6.2 to make the strategy of the proof easy to catch. By Corollary 5.3 proved by Krone, the term $1-\pi\left(A, B, \frac{\lambda}{2 d}, d\right)$ is 
the survival probability of types 1 and 2 vertices of the 'on-off' process $\left\{\xi_{t}^{(B, A)}\right\}_{t \geq 0}$. By Lemma 5.4, the term

$$
1-\left(\frac{1+\delta+\gamma}{\frac{(2 d-M) \lambda}{2 d} \gamma}\right)^{n}\left(1-\frac{\frac{(2 d-M) \lambda}{2 d} \gamma-(1+\delta+\gamma)}{\frac{(2 d-M) \lambda}{2 d}(\gamma+1)}\right)^{m}
$$

is the survival probability of the two-type branching process with parameters $\frac{2 d-M}{2 d} \lambda, \gamma, \delta$ and $n$ type 2 individuals and $m$ type 1 individuals at $t=0$, which we will show in the proof that is a lower bound of the probability that the number of types 1 and 2 vertices of $\left\{\xi_{t}^{(B, A)}\right\}_{t \geq 0}$ reaches $M$ for some $t \geq 0$. We will show in the proof that the term $\widetilde{\alpha}(M)$ is a lower bound of the probability that the number of type 2 vertices of the 'on-off' process $\left\{\xi_{t}\right\}_{t \geq 0}$ reaches $\left[\frac{M e^{-(1+\delta)}\left(1-e^{-\gamma}\right)}{2}\right]$ for some $t \geq 0$ conditioned on there being at least $M$ types 1 and 2 vertices at $t=0$. We will show in the proof that the term $\widetilde{b}\left(d,\left\lceil\frac{M e^{-(1+\delta)}\left(1-e^{-\gamma}\right)}{2}\right], \lambda\right)$ is a lower bound of the probability that types 1 and 2 vertices of the 'on-off' process $\left\{\xi_{t}\right\}_{t \geq 0}$ survives conditioned on there being $\left\lceil\frac{M e^{-(1+\delta)}\left(1-e^{-\gamma}\right)}{2}\right]$ type 2 vertices at $t=0$. According to the above meanings of the four terms, Lemma 6.2 holds according to the strong Markov property. Now we give the details of the proof of Lemma 6.2.

Proof of Lemma 6.2: Throughout this proof we assume that $n, m, M, d$ are fixed such that $M>n+m$ and $2 d>M$. For $A, B \subseteq \mathbb{Z}^{d}$ such that $|A|=m,|B|=n$ and $A \cap B=\emptyset$, let

$$
\tau_{M}(A, B)=\inf \left\{t \geq 0:\left|\widehat{C}_{t}^{(B, A)}\right|+\left|\widehat{D}_{t}^{(B, A)}\right|=M\right\}
$$

where $\left\{\xi_{t}\right\}_{t \geq 0}$ is the 'on-off' process introduced in Section 5 and

$$
\widehat{C}_{t}=\left\{x: \xi_{t}(x)=2\right\} \text { while } \widehat{D}_{t}=\left\{x: \xi_{t}(x)=1\right\}
$$

defined as in Section 5. Let $\left\{\left(\widehat{\zeta}_{t}^{M}, \widehat{g}_{t}^{M}\right)\right\}_{t \geq 0}$ be the two-type branching process defined as in Section 5 with parameter $\frac{2 d-\bar{M}}{2 d} \lambda, \gamma, \delta$, then we define

$$
\widehat{\tau}_{M}=\inf \left\{t \geq 0: \widehat{\zeta}_{t}^{M}+\widehat{g}_{t}^{M}=M\right\} .
$$

For the 'on-off' process $\left\{\xi_{t}^{(B, A)}\right\}_{t \geq 0}$ on $\mathbb{Z}^{d}$ with parameter $\frac{\lambda}{2 d}$, a type 2 vertex gives birth to a type 1 vertex at rate at least

$$
\frac{\lambda}{2 d} \times(2 d-M)
$$

before the moment $\tau_{M}(A, B)$, since there are at lest $(2 d-M)$ neighbors in state 0 before the moment $\tau_{M}(A, B)$. As a result, $\left|\widehat{C}_{t}^{(B, A)}\right|+\left|\widehat{D}_{t}^{(B, A)}\right|$ is stochastically dominated from below by $\widehat{\zeta}_{t}^{M}+\widehat{g}_{t}^{M}$ with $\widehat{\zeta}_{0}^{M}=n$ and $\widehat{g}_{0}^{M}=m$ for $t \in$ $\left[0, \tau_{M}(A, B)\right)$. In detail, the Vasershtein coupling of $\left(\left|\widehat{C}_{t}^{(B, A)}\right|,\left|\widehat{D}_{t}^{(B, A)}\right|\right)$ and $\left(\widehat{\zeta}_{t}^{M}, \widehat{g}_{t}^{M}\right)$ for $0 \leq t \leq \tau_{M}(A, B)$ is given as follows. For any $0 \leq t \leq \tau_{M}(A, B)$, 
$\left(\left|\widehat{C}_{t}^{(B, A)}\right|,\left|\widehat{D}_{t}^{(B, A)}\right|, \widehat{\zeta}_{t}^{M}, \widehat{g}_{t}^{M}\right)$ flips to

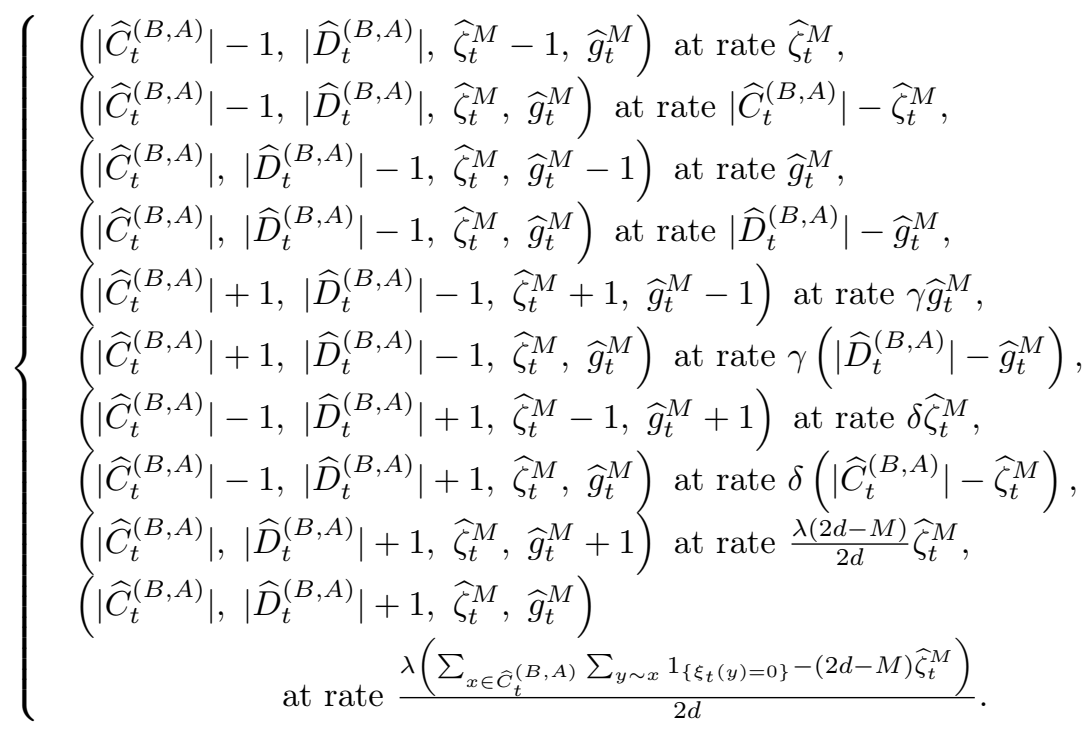

Note that the above coupling maintains the property that $\left|\widehat{C}_{t}^{(B, A)}\right| \geq \widehat{\zeta}_{t}^{M}$ and $\left|\widehat{D}_{t}^{(B, A)}\right| \geq \widehat{g}_{t}^{M}$ for $0 \leq t \leq \tau_{M}(A, B)$. Therefore,

$$
P_{\frac{\lambda}{2 d}, d}\left(\tau_{M}(A, B)<+\infty\right) \geq P\left(\widehat{\tau}_{M}<+\infty \mid \widehat{\zeta}_{0}^{M}=n, \widehat{g}_{0}^{M}=m\right) .
$$

We claim that

$$
\begin{aligned}
& P\left(\widehat{\tau}_{M}<+\infty \mid \widehat{\zeta}_{0}^{M}=n, \widehat{g}_{0}^{M}=m\right) \geq \\
& P\left(\widehat{\zeta}_{t}^{M}+\widehat{g}_{t}^{M}>0 \text { for all } t \geq 0 \mid \widehat{\zeta}_{0}^{M}=n, \widehat{g}_{0}^{M}=m\right) .
\end{aligned}
$$

Equation (6.13) holds according to the following analysis. Let

$$
\widetilde{\Xi}=\inf \left\{P\left(\widehat{\zeta}_{\frac{1}{2}}^{M}+\widehat{g}_{\frac{1}{2}}^{M}=0 \mid \widehat{\zeta}_{0}^{M}=k, \widehat{g}_{0}^{M}=l\right): l+k \leq M\right\},
$$

then $\widetilde{\Xi}>0$ since $P\left(\widehat{\zeta}_{\frac{1}{2}}^{M}+\widehat{g}_{\frac{1}{2}}^{M}=0 \mid \widehat{\zeta}_{0}^{M}=k, \widehat{g}_{0}^{M}=l\right)>0$ for each pair of $(l, k)$ and there are finite many pairs of $(l, k) \mathrm{s}$ satisfying $l+k \leq M$. Let

$$
\widetilde{\tau}=\inf \left\{n: n \text { is a nonnegative integer and } \widehat{\zeta}_{n+\frac{1}{2}}^{M}+\widehat{g}_{n+\frac{1}{2}}^{M}=0\right\},
$$

then $\widehat{\zeta}_{n}^{M}+\widehat{g}_{n}^{M} \leq M$ for each integer $n \geq 0$ on the event $\left\{\widehat{\tau}_{M}=+\infty\right\}$ and hence $\widetilde{\tau}$ is stochastically dominated from above by the geometric random variable $\widetilde{Y}$ satisfying

$$
P(\widetilde{Y}=n)=\widetilde{\Xi}(1-\widetilde{\Xi})^{n}
$$

for $n=0,1,2, \ldots$ on the event $\left\{\widehat{\tau}_{M}=+\infty\right\}$. As a result,

$$
P\left(\widetilde{\tau}<+\infty \mid \widehat{\tau}_{M}=+\infty\right) \geq P(\tilde{Y}<+\infty)=1 .
$$

That is to say

$$
\left\{\widehat{\zeta}_{t}^{M}+\widehat{g}_{t}^{M}=0 \text { for some } t \geq 0\right\} \supseteq\left\{\widehat{\tau}_{M}=+\infty\right\}
$$


in the sense of ignoring a set with probability zero, Equation (6.13) follows from which directly.

By Lemma 5.4 with $\lambda$ replaced by $\frac{2 d-M}{2 d} \lambda$,

$$
\begin{aligned}
& P\left(\widehat{\zeta}_{t}^{M}+\widehat{g}_{t}^{M}>0 \text { for all } t \geq 0 \mid \widehat{\zeta}_{0}^{M}=n, \widehat{g}_{0}^{M}=m\right) \\
& =1-\left(\frac{1+\delta+\gamma}{\frac{(2 d-M) \lambda}{2 d} \gamma}\right)^{n}\left(1-\frac{\frac{(2 d-M) \lambda}{2 d} \gamma-(1+\delta+\gamma)}{\frac{(2 d-M) \lambda}{2 d}(\gamma+1)}\right)^{m} .
\end{aligned}
$$

Then, by Equations (6.12) and (6.13),

$$
\begin{aligned}
& P_{\frac{\lambda}{2 d}, d}\left(\tau_{M}(A, B)<+\infty\right) \\
& \geq 1-\left(\frac{1+\delta+\gamma}{\frac{(2 d-M) \lambda}{2 d} \gamma}\right)^{n}\left(1-\frac{\frac{(2 d-M) \lambda}{2 d} \gamma-(1+\delta+\gamma)}{\frac{(2 d-M) \lambda}{2 d}(\gamma+1)}\right)^{m} .
\end{aligned}
$$

We claim that the 'on-off' process is also monotonic with respect to the partial order ' $\preceq$ ' defined in Section 2. To prove this claim, we couple two 'on-off' processes $\left\{\xi_{t}^{(1)}\right\}_{t \geq 0}$ and $\left\{\xi_{t}^{(2)}\right\}_{t \geq 0}$ with initial condition $\xi_{0}^{(2)} \preceq \xi_{0}^{(1)}$ in the following way, which is also a Vasershtein coupling. For any $t \geq 0$ and each $x \in \mathbb{Z}^{d},\left(\xi_{t}^{(1)}(x), \xi_{t}^{(2)}(x)\right)$ flips from $(i, j)$ to $(l, k)$ at rate

$$
\begin{cases}\delta & \text { if }(i, j)=(2,2) \text { and }(l, k)=(1,1), \\ 1 & \text { if }(i, j)=(2,2) \text { and }(l, k)=(0,0), \\ \delta & \text { if }(i, j)=(2,1) \text { and }(l, k)=(1,1), \\ 1 & \text { if }(i, j)=(2,1) \text { and }(l, k)=(0,0), \\ \gamma & \text { if }(i, j)=(2,1) \text { and }(l, k)=(2,2), \\ \delta & \text { if }(i, j)=(2,0) \text { and }(l, k)=(1,0), \\ 1 & \text { if }(i, j)=(2,0) \text { and }(l, k)=(0,0), \\ \lambda \sum_{y \sim x} 1_{\left\{\xi_{t}^{(2)}(y)=2\right\}} & \text { if }(i, j)=(2,0) \text { and }(l, k)=(2,1), \\ \gamma & \text { if }(i, j)=(1,1) \text { and }(l, k)=(2,2), \\ 1 & \text { if }(i, j)=(1,1) \text { and }(l, k)=(0,0), \\ \gamma & \text { if }(i, j)=(1,0) \text { and }(l, k)=(2,0), \\ 1 & \text { if }(i, j)=(1,0) \text { and }(l, k)=(0,0), \\ \lambda \sum_{y \sim x} 1_{\left\{\xi_{t}^{(2)}(y)=2\right\}} & \text { if }(i, j)=(1,0) \text { and }(l, k)=(1,1), \\ \lambda \sum_{y \sim x} 1_{\left\{\xi_{t}^{(2)}(y)=2\right\}} & \text { if }(i, j)=(0,0) \text { and }(l, k)=(1,1), \\ \lambda \sum_{y \sim x}\left(1_{\left\{\xi_{t}^{(1)}(y)=2\right\}}-1_{\left\{\xi_{t}^{(2)}(y)=2\right\}}\right) & \text { if }(i, j)=(0,0) \text { and }(l, k)=(1,0) .\end{cases}
$$

Note that the above coupling maintains the property that $\xi_{t}^{(2)} \preceq \xi_{t}^{(1)}$ for all $t \geq 0$. As a result, for $A_{1}, B_{1} \subseteq \mathbb{Z}^{d}$ such that $A_{1} \cap B_{1}=\emptyset$ while $\left|A_{1}\right|+\left|B_{1}\right|=M$,

$$
\begin{aligned}
& P_{\frac{\lambda}{2 d}, d}\left(\widehat{C}_{t}^{\left(B_{1}, A_{1}\right)} \cup \widehat{D}_{t}^{\left(B_{1}, A_{1}\right)} \neq \emptyset \text { for all } t \geq 0\right) \geq \\
& P_{\frac{\lambda}{2 d}, d}\left(\widehat{C}_{t}^{\left(\emptyset, A_{1} \cup B_{1}\right)} \cup \widehat{D}_{t}^{\left(\emptyset, A_{1} \cup B_{1}\right)} \neq \emptyset \text { for all } t \geq 0\right) .
\end{aligned}
$$


By direct calculation, an initial type 1 vertex becomes a type 2 vertex at some moment $s<1$ and then stays in state 2 till moment $t=1$ with probability at least

$$
P\left(\widetilde{Y}_{2}>1, \widetilde{Y}_{3}<1, \widetilde{Y}_{4}>1\right)=e^{-(1+\delta)}\left(1-e^{-\gamma}\right),
$$

where $\widetilde{Y}_{2}, \widetilde{Y}_{3}, \widetilde{Y}_{4}$ are independent exponential times with rates $1, \gamma, \delta$ respectively. Therefore, for $A_{1}, B_{1} \subseteq \mathbb{Z}^{d}$ such that $A_{1} \cap B_{1}=\emptyset$ while $\left|A_{1}\right|+\left|B_{1}\right|=M,\left|\widehat{C}_{1}^{\left(\emptyset, A_{1} \cup B_{1}\right)}\right|$ is stochastically dominated from below by a random variable following the binomial distribution $B\left(M, e^{-(1+\delta)}\left(1-e^{-\gamma}\right)\right)$ and

$$
P_{\frac{\lambda}{2 d}, d}\left(\left|\widehat{C}_{1}^{\left(\emptyset, A_{1} \cup B_{1}\right)}\right| \geq \frac{M e^{-(1+\delta)}\left(1-e^{-\gamma}\right)}{2}\right) \geq \widetilde{\alpha}(M) .
$$

For $A_{2}, B_{2} \subseteq \mathbb{Z}^{d}$ such that $\left|B_{2}\right|=\left\lceil\frac{M e^{-(1+\delta)}\left(1-e^{-\gamma}\right)}{2}\right\rceil$ and $A_{2} \cap B_{2}=\emptyset$, by Corollary 5.3 and the definition of $\widetilde{b}\left(d,\left\lceil\frac{M e^{-(1+\delta)}\left(1-e^{-\gamma}\right)}{2}\right\rceil, \lambda\right)$,

$$
\begin{aligned}
& P_{\frac{\lambda}{2 d}, d}\left(\widehat{C}_{t}^{\left(B_{2}, A_{2}\right)} \cup \widehat{D}_{t}^{\left(B_{2}, A_{2}\right)} \neq \emptyset \text { for all } t \geq 0\right) \\
& \geq P_{\frac{\lambda}{2 d}, d}\left(\widehat{C}_{t}^{\left(B_{2}, \emptyset\right)} \cup \widehat{D}_{t}^{\left(B_{2}, \emptyset\right)} \neq \emptyset \text { for all } t \geq 0\right) \\
& =1-\pi\left(\emptyset, B_{2}, \frac{\lambda}{2 d}, d\right)=\nu_{\frac{\lambda}{2 d}, d}\left(\eta(x) \neq 0 \text { for some } x \in B_{2}\right) \\
& \geq \widetilde{b}\left(d,\left\lceil\frac{M e^{-(1+\delta)}\left(1-e^{-\gamma}\right)}{2}\right\rceil, \lambda\right) .
\end{aligned}
$$

By Equations (6.16), (6.17) and the Markov property, for $A_{1}, B_{1} \subseteq \mathbb{Z}^{d}$ such that $A_{1} \cap B_{1}=\emptyset$ while $\left|A_{1}\right|+\left|B_{1}\right|=M$,

$$
\begin{aligned}
& P_{\frac{\lambda}{2 d}, d}\left(\widehat{C}_{t}^{\left(B_{1}, A_{1}\right)} \cup \widehat{D}_{t}^{\left(B_{1}, A_{1}\right)} \neq \emptyset \text { for all } t \geq 0\right) \\
& \geq \widetilde{\alpha}(M) \widetilde{b}\left(d,\left\lceil\frac{M e^{-(1+\delta)}\left(1-e^{-\gamma}\right)}{2}\right\rceil, \lambda\right) .
\end{aligned}
$$

On the event $\tau_{M}(A, B)<+\infty, \widetilde{B}:=\widehat{C}_{\tau_{M}(A, B)}^{(B, A)}$ and $\widetilde{A}:=\widehat{D}_{\tau_{M}(A, B)}^{(B, A)}$ satisfy $\widetilde{A} \cap \widetilde{B}=\emptyset$ while $|\widetilde{A}|+|\widetilde{B}|=M$. Therefore, by Equations (6.14), (6.18) and the strong Markov property,

$$
\begin{aligned}
& P_{\frac{\lambda}{2 d}, d}\left(\widehat{C}_{t}^{(B, A)} \cup \widehat{D}_{t}^{(B, A)} \neq \emptyset \text { for all } t \geq 0\right) \\
& \geq\left\{1-\left(\frac{1+\delta+\gamma}{\frac{(2 d-M) \lambda}{2 d} \gamma}\right)^{n}\left(1-\frac{\frac{(2 d-M) \lambda}{2 d} \gamma-(1+\delta+\gamma)}{\frac{(2 d-M) \lambda}{2 d}(\gamma+1)}\right)^{m}\right\} \\
& \times \widetilde{\alpha}(M) \widetilde{b}\left(d,\left\lceil\frac{M e^{-(1+\delta)}\left(1-e^{-\gamma}\right)}{2}\right\rceil, \lambda\right)
\end{aligned}
$$

for $A, B \subseteq \mathbb{Z}^{d}$ such that $|A|=m,|B|=n$ and $A \cap B=\emptyset$. Lemma 6.2 follows from Corollary 5.3 and Equation (6.19) directly.

\section{Acknowledgements}

The author is grateful to the reviewers. Their comments are great help for the improvement of this paper. The author is grateful to the financial support from the National Natural Science Foundation of China with grant number 11501542. 


\section{References}

Durrett, R. Probability: theory and examples, volume 31 of Cambridge Series in Statistical and Probabilistic Mathematics. Cambridge University Press, Cambridge, fourth edition (2010). ISBN 978-0-521-76539-8. MR2722836.

Foxall, E. New results for the two-stage contact process. J. Appl. Probab., 52 (1), 258-268 (2015). MR3336860.

Griffeath, D. The binary contact path process. Ann. Probab., 11 (3), 692-705 (1983). MR704556.

Harris, T. E. Contact interactions on a lattice. Ann. Probability, 2, 969-988 (1974). MR356292.

Harris, T. E. On a class of set-valued Markov processes. Ann. Probability, 4 (2), 175-194 (1976). MR0400468.

Harris, T. E. Additive set-valued Markov processes and graphical methods. Ann. Probab., 6 (3), 355-378 (1978). MR0488377.

Holley, R. and Liggett, T. M. Generalized potlatch and smoothing processes. Z Wahrsch. Verw. Gebiete, 55 (2), 165-195 (1981). MR608015.

Kesten, H. On the number of self-avoiding walks. II. J. Mathematical Phys., 5, 1128-1137 (1964). MR166845.

Kesten, H. Asymptotics in high dimensions for percolation. In Disorder in physical systems, Oxford Sci. Publ., pp. 219-240. Oxford Univ. Press, New York (1990). MR1064563.

Krone, S. M. The two-stage contact process. Ann. Appl. Probab., 9 (2), 331-351 (1999). MR1687347.

Lang, S. Undergraduate analysis. Undergraduate Texts in Mathematics. SpringerVerlag, New York (1983). ISBN 0-387-90800-5. MR704509.

Liggett, T. M. Interacting particle systems, volume 276 of Grundlehren der Mathematischen Wissenschaften [Fundamental Principles of Mathematical Sciences]. Springer-Verlag, New York (1985). ISBN 0-387-96069-4. MR776231.

Liggett, T. M. Stochastic interacting systems: contact, voter and exclusion processes, volume 324 of Grundlehren der Mathematischen Wissenschaften [Fundamental Principles of Mathematical Sciences]. Springer-Verlag, Berlin (1999). ISBN 3-540-65995-1. MR1717346.

Schonmann, R. H. and Vares, M. E. The survival of the large-dimensional basic contact process. Probab. Theory Relat. Fields, 72 (3), 387-393 (1986). MR843501.

van der Hofstad, R. Random graphs and complex networks. Vol. 1. Cambridge Series in Statistical and Probabilistic Mathematics, [43]. Cambridge University Press, Cambridge (2017). ISBN 978-1-107-17287-6. MR3617364.

$\mathrm{Xue}, \mathrm{X}$. The critical infection rate of the high-dimensional two-stage contact process. Statist. Probab. Lett., 140, 115-125 (2018). MR3812258. 